\title{
Highly Efficient Synthesis and Solid-State Characterization of 1,2,4,5-Tetrakis(alkyl- and arylamino)benzenes and Cyclization to their Respective Benzobis(imidazolium) Salts
}

\author{
Dimitri M. Khramov, Andrew. J. Boydston, and Christopher W. Bielawski* \\ The University of Texas at Austin, Department of Chemistry and Biochemsitry, \\ Austin, TX 78712 USA; email: bielawski@cm.utexas.edu
}

\section{SUPPORTING INFORMATION}

General Considerations: All reactions were conducted under an atmosphere of nitrogen using standard Schlenk techniques or in a nitrogen-filled glove-box. $\mathrm{CHCl}_{3}$ was degassed by two freeze-pump-thaw cycles and stored over $4 \AA$ molecular sieves and $\mathrm{K}_{2} \mathrm{CO}_{3}$. Hexanes were distilled from $\mathrm{CaH}_{2}$ and degassed by two freeze-pump-thaw cycles. THF and toluene were freshly distilled from $\mathrm{Na}$ /benzophenone and degassed by two freeze-pump-thaw cycles. All reagents were purchased from Aldrich or Acros and were used without further purification. ${ }^{1} \mathrm{H}$ NMR spectra were recorded using a Varian Gemini $(300 \mathrm{MHz}$ or $400 \mathrm{MHz}$ ) spectrometer. Chemical shifts are reported in delta $(\delta)$ units, expressed in parts per million $(\mathrm{ppm})$ downfield from tetramethylsilane using the residual protonated solvent as an internal standard $\left(\mathrm{CDCl}_{3}, 7.24\right.$ ppm; $\mathrm{C}_{6} \mathrm{D}_{6}, 7.15 \mathrm{ppm}$; DMSO- $\left.d_{6}, 2.49 \mathrm{ppm}\right) .{ }^{13} \mathrm{C}$ NMR spectra were recorded using a Varian Gemini $(100 \mathrm{MHz})$ spectrometer. Chemical shifts are reported in delta $(\delta)$ units, expressed in parts per million (ppm) downfield from tetramethylsilane using the solvent as an internal standard $\left(\mathrm{CDCl}_{3}, 77.0 \mathrm{ppm} ; \mathrm{C}_{6} \mathrm{D}_{6}, 128 \mathrm{ppm} ; \mathrm{DMSO}-d_{6}, 39.5 \mathrm{ppm}\right) .{ }^{13} \mathrm{C}$ NMR spectra were routinely run with broadband decoupling. High-resolution mass spectra (HRMS) were obtained with a VG analytical ZAB2-E or a Karatos MS9 instrument and are reported as $\mathrm{m} / \mathrm{z}$ (relative intensity).

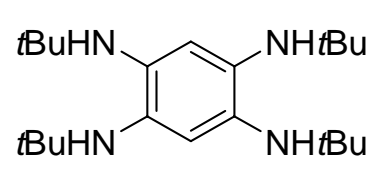

1,2,4,5-Tetrakis-N-tert-butylaminobenzene (4). A catalyst for mediating aryl-amination coupling reaction was prepared by charging a $20 \mathrm{~mL}$ vial with 1,3-bis(2,6-diisopropylphenyl)imidazolium chloride ( $0.085 \mathrm{~g}, 0.2$ $\mathrm{mmol}), \mathrm{NaOtBu}(0.03 \mathrm{~g}, 0.3 \mathrm{mmol}), \mathrm{Pd}(\mathrm{OAc})_{2}(0.025 \mathrm{~g}, 0.1 \mathrm{mmol})$, toluene $(5 \mathrm{~mL})$, and a stir bar followed by stirring this mixture at $80{ }^{\circ} \mathrm{C}$ for 10 minutes. The catalyst solution was added to a $250 \mathrm{~mL}$ flask containing $1,2,4,5$-tetrabromobenzene $(2.22 \mathrm{~g}$, $5.64 \mathrm{mmol})$ suspended in toluene $(100 \mathrm{~mL})$. tert-Butyl amine $(1.73 \mathrm{~g}, 23.7 \mathrm{mmol})$ and $\mathrm{NaOtBu}$ $(2.65 \mathrm{~g}, 27.6 \mathrm{mmol})$ were then added and the resulting mixture was sealed and stirred at $110^{\circ} \mathrm{C}$ overnight. After cooling to ambient temperature, precipitated solids were collected by filtration under a cone of nitrogen, dissolved in degassed $\mathrm{CHCl}_{3}(100 \mathrm{~mL})$, and filtered to remove residual $\mathrm{NaBr}$ salts. Solvent was then removed under reduced pressure to afford $1.96 \mathrm{~g} \mathrm{(96 \%} \mathrm{yield)} \mathrm{of} \mathrm{the}$ desired product as a gray solid. Note: the product slowly oxidizes to the corresponding benzoquinonediimine over several days in aerated solutions. As a solid, the product is bench stable for >months. ${ }^{1} \mathrm{H}$ NMR $\left(\mathrm{CDCl}_{3}\right): \delta 6.53(\mathrm{~s}, 2 \mathrm{H}), 3.73(\mathrm{br}, 4 \mathrm{H}), 1.22(\mathrm{~s}, 36 \mathrm{H}) .{ }^{13} \mathrm{C}$ NMR $\left(\mathrm{CDCl}_{3}\right): \delta 131.7,115.5,52.0$, 30.0. HRMS: $[\mathrm{M}]^{+}$calcd for $\mathrm{C}_{22} \mathrm{H}_{42} \mathrm{~N}_{4}: 362.3409$; Found, 362.3406 . 


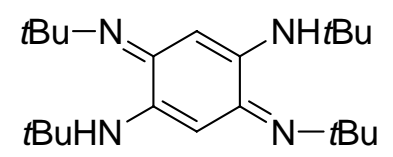

$\mathrm{N}, \mathrm{N}^{\prime}, \mathrm{N}^{\prime \prime}, \mathrm{N}^{\prime \prime}$-tetra(tert-butyl)-2,5-diamino-1,4-benzoquinonediimine (5). Compound $4(0.1 \mathrm{~g}, 0.28 \mathrm{mmol})$ was dissolved in $\mathrm{CHCl}_{3}(3 \mathrm{~mL})$ and stirred under a ballon of oxygen for $24 \mathrm{~h}$ followed by removal of solvent to obtain a yellow powder $(0.1 \mathrm{~g})$ in quantitative yield. Alternatively, oxidations can be accomplished in less than 5 minutes by adding an excess of $\mathrm{H}_{2} \mathrm{O}_{2}$. ${ }^{1} \mathrm{H}$ NMR $\left(\mathrm{CDCl}_{3}\right): \delta 5.53(\mathrm{~s}, 2 \mathrm{H}), 1.35(\mathrm{~s}, 36 \mathrm{H}) \cdot{ }^{13} \mathrm{C}$ NMR $\left(\mathrm{CDCl}_{3}\right): \delta 138,90.8,52.0,29.8$. HRMS: $[\mathrm{M}]^{+}$ calcd for $\mathrm{C}_{24} \mathrm{H}_{41} \mathrm{~N}_{4}$ : 361.3331 ; Found: 361.3326 .

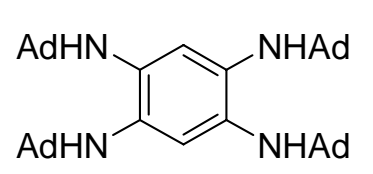

1,2,4,5-Tetrakis- $\mathrm{N}$-adamantylaminobenzene (6). The aryl amination was performed in a manner analogous to the synthesis of $\mathbf{4}$ using 1,2,4,5tetrabromobenzene $(13.97 \mathrm{~g}, 35.5 \mathrm{mmol})$ and adamantyl amine $(22.5 \mathrm{~g}$, 149 mol). Upon completion, the cooled reaction mixture was filtered in a drybox to afford a beige solid. The solids were rinsed with toluene and extracted with degassed $\mathrm{CHCl}_{3}$ and the extract was concentrated under reduced pressure to afford $21.5 \mathrm{~g}$ ( $90 \%$ yield) of the desired product as a white powder. Note: the product slowly oxidizes to the corresponding benzoquinone-diimine over several days in aerated solutions. As a solid, the product is bench stable for $>$ months. ${ }^{1} \mathrm{H}$ NMR $\left(\mathrm{CDCl}_{3}\right): \delta 6.59(\mathrm{~s}, 2 \mathrm{H}), 2.06(\mathrm{br}, 12 \mathrm{H}), 1.81(\mathrm{br}, 24 \mathrm{H}), 1.66-1.58$ (br, 24H). ${ }^{13} \mathrm{C}$ NMR $\left(\mathrm{CDCl}_{3}\right): \delta 131.3,117.8,52.5,43.7,36.6,29.8$. HRMS: $[\mathrm{M}]^{+}$calcd for $\mathrm{C}_{46} \mathrm{H}_{66} \mathrm{~N}_{4}, 674.5287$; Found, 674.5286 .

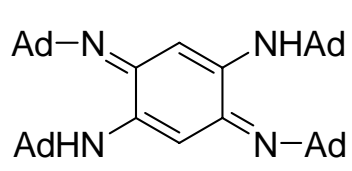

$\mathbf{N}, \mathbf{N}^{\prime}, \mathrm{N}^{\prime \prime}, \mathrm{N}^{\prime \prime \prime}$-tetra(adamantyl)-2,5-diamino-1,4-benzoquinonediimine. Compound $4(0.1 \mathrm{~g}, 0.15 \mathrm{mmol})$ was dissolved in $\mathrm{CH}_{2} \mathrm{Cl}_{2}(3 \mathrm{~mL})$ and stirred under a ballon of oxygen for $24 \mathrm{~h}$ followed by removal of solvent to obtain a yellow powder $(0.1 \mathrm{~g})$ in quantitative yield. ${ }^{1} \mathrm{H}$ NMR $\left(\mathrm{CDCl}_{3}\right): \delta 6.69(\mathrm{br}, 2 \mathrm{H}), 5.77(\mathrm{~s}, 2 \mathrm{H}), 2.10(\mathrm{br}, 12 \mathrm{H}), 1.97(\mathrm{br}, 24 \mathrm{H}), 1.68(\mathrm{br}, 24 \mathrm{H}) .{ }^{13} \mathrm{C} \mathrm{NMR}$ $\left(\mathrm{CDCl}_{3}\right): \delta 138,92.2,52.7,42.6,36.9,29.8$. HRMS: $[\mathrm{M}+\mathrm{H}]^{+}$calcd for $\mathrm{C}_{46} \mathrm{H}_{64} \mathrm{~N}_{4}, 673.5209$; Found, 673.5209 .

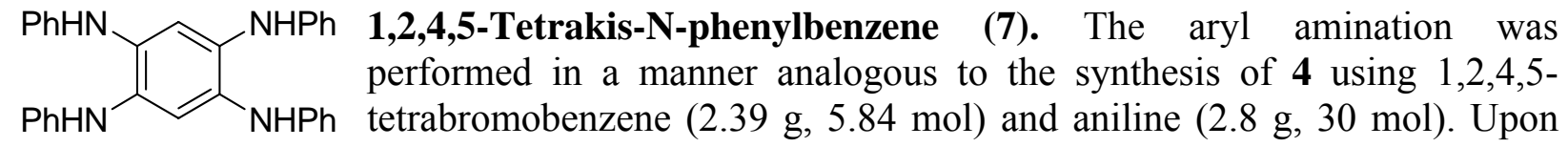
completion, the cooled reaction mixture was filtered in a drybox to afford a beige solid. The solids were rinsed with excess hexanes and extracted with degassed $\mathrm{CHCl}_{3}$. The extract was then concentrated under reduced pressure to afford $1.78 \mathrm{~g}(76 \%)$ of the desired product as a light pink powder. ${ }^{1} \mathrm{H}$ NMR $\left(\mathrm{CDCl}_{3}\right): \delta$ 7.24-7.19 (br m, 12H), 6.9-6.84 (br, $\left.12 \mathrm{H}\right), 5.56(\mathrm{~s}, 2 \mathrm{H}) .{ }^{13} \mathrm{C}$ NMR $\left(\mathrm{CDCl}_{3}\right): \delta$ 144.5, 130.6, 129.3, 120.2, 116.6, 113.9. HRMS: [M] ${ }^{+}$calcd for $\mathrm{C}_{30} \mathrm{H}_{25} \mathrm{~N}_{4}, 441.2074$; Found: 441.2074 .

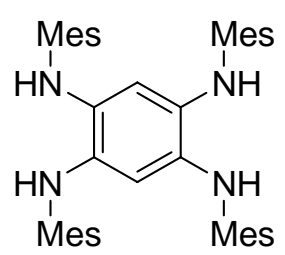

1,2,4,5-Tetrakis(2,4,6-trimethylphenylamino)benzene (8). The aryl amination was performed in a manner analogous to the synthesis of $\mathbf{4}$ using 1,2,4,5-tetrabromobenzene $(2.00 \mathrm{~g}, 5.1 \mathrm{mmol})$ and mesityl amine $(7 \mathrm{~mL}, 51$ $\mathrm{mmol})$. Upon completion, the cooled reaction mixture was filtered in a drybox to afford a beige solid. The solids were rinsed with toluene and extracted with degassed $\mathrm{CHCl}_{3}$. The extract was then concentrated under reduced pressure to afford $2.71 \mathrm{~g}(94 \%)$ of the desired product as a white powder. ${ }^{1} \mathrm{H} \mathrm{NMR}\left(\mathrm{CDCl}_{3}\right): \delta 6.75(\mathrm{~s}, 8 \mathrm{H})$, $5.40(\mathrm{~s}, 2 \mathrm{H}), 4.86$ (br s, 4H), $2.22(\mathrm{~s}, 12 \mathrm{H}), 2.05$ (s, $24 \mathrm{H}) .{ }^{13} \mathrm{C} \mathrm{NMR}\left(\mathrm{CDCl}_{3}\right): \delta 137.9,132.6$, 
132.1, 129.4, 129.0, 105.3, 20.6, 18.2. HRMS: $[\mathrm{M}]^{+}$calcd for $\mathrm{C}_{42} \mathrm{H}_{49} \mathrm{~N}_{4}, 609.3978$; Found: 609.3952 .

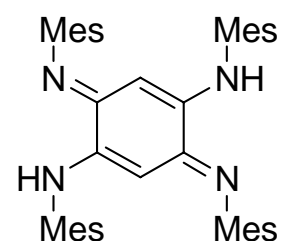

Mesityl-derived azophenine (9). The tetraamine $8(0.1 \mathrm{~g}, 0.164 \mathrm{mmol})$ was stirred in aerated $\mathrm{CHCl}_{3}(3 \mathrm{~mL})$ for $2 \mathrm{~h}$. The mixture was concentrated to give an orange-red solid $(0.1 \mathrm{~g})$ in quantitative yield. ${ }^{1} \mathrm{H}$ NMR $\left(\mathrm{CDCl}_{3}\right): \delta 6.79(\mathrm{~s}$, $4 \mathrm{H}), 6.75(\mathrm{~s}, 4 \mathrm{H}), 5.40(\mathrm{~s}, 2 \mathrm{H}), 4.61(\mathrm{~s}, 2 \mathrm{H}), 2.23(\mathrm{~s}, 12 \mathrm{H}), 2.07(\mathrm{~s}, 12 \mathrm{H}), 2.05$
$(\mathrm{~s}, 12 \mathrm{H}) .{ }^{13} \mathrm{C} \mathrm{NMR}\left(\mathrm{CDCl}_{3}\right): \delta 137.9,132.6,132.1,129.4,129.0,128.6,88.9$, 20.8, 20.6, 18.2, 18.0. HRMS: [M] calcd for $\mathrm{C}_{42} \mathrm{H}_{49} \mathrm{~N}_{4}, 609.3956$; Found: 609.3952.

o-An o-An 1,2,4,5-Tetrakis(2-methoxyphenylamino)benzene (10). The aryl amination $\mathrm{NH}$ was performed in a manner analogous to the synthesis of 4 using 1,2,4,5-

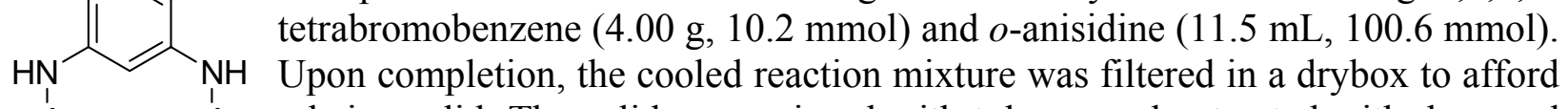
o-An o-An a beige solid. The solids were rinsed with toluene and extracted with degassed $\mathrm{CHCl}_{3}$ and the extract was concentrated under reduced pressure to afford $6.92 \mathrm{~g}(96 \%)$ of the desired product as an off-white powder. Alternatively, the cooled reaction mixture was opened under a cone of nitrogen and $\mathrm{HCl}(2 \mathrm{~mL})$ in $\mathrm{MeOH}(10 \mathrm{~mL})$ was added quickly to produce a deep purple slurry. The mixture was poured into $\mathrm{H}_{2} \mathrm{O}(25 \mathrm{~mL})$ and the solids were collected via vacuum filtration, rinsed with $\mathrm{H}_{2} \mathrm{O}$ and $\mathrm{Et}_{2} \mathrm{O}$, and dried under vacuum to provide the tetrahydrochloride salt as a light purple solid in nearly quantitative yield. ${ }^{1} \mathrm{H}$ NMR of free base $\left(\mathrm{CDCl}_{3}\right): \delta 7.33(\mathrm{~s}, 2 \mathrm{H}), 7.06-7.04(\mathrm{~m}, 4 \mathrm{H}), 6.86-6.76(\mathrm{~m}, 12 \mathrm{H}), 6.02(\mathrm{br} \mathrm{s}, 4 \mathrm{H}), 3.82(\mathrm{~s}, 12 \mathrm{H})$. ${ }^{13} \mathrm{C}$ NMR $\left(\mathrm{CDCl}_{3}\right): \delta 148.2,134.4,130.5,121.0,119.1,114.4,110.3,114.2,55.5$. HRMS: $[\mathrm{M}]^{+}$ calcd for $\mathrm{C}_{34} \mathrm{H}_{35} \mathrm{~N}_{4} \mathrm{O}_{4}, 563.2606$; Found 563.2653.

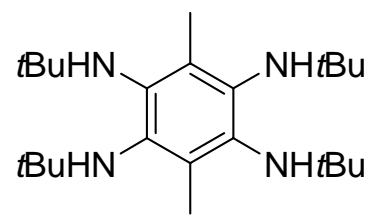

1,2,4,5-Tetrakis(tert-butylamino)-para-xylene (11). The aryl amination was performed in a manner analogous to the synthesis of 4 using 2,3,5,6tetrabromoxylene $(3.0 \mathrm{~g}, 7.11 \mathrm{~mol})$ and tert-butyl amine $(2.13 \mathrm{~g}, 29.2$ mmol). Upon completion, the cool reaction mixture was filtered over Celite under a cone of nitrogen with the aid of degassed toluene. The filtrate was concentrated under reduced pressure to afford $2.7 \mathrm{~g}(99 \%)$ of the desired product as a brown solid. It should be noted that the product is bench stable in aerated solutions for $>$ months. ${ }^{1} \mathrm{H}$ NMR $\left(\mathrm{CDCl}_{3}\right): \delta 3.39(\mathrm{br}, 4 \mathrm{H}), 2.28(\mathrm{~s}, 6 \mathrm{H}), 1.06(\mathrm{~s}, 36 \mathrm{H}) .{ }^{13} \mathrm{C}$ NMR $\left(\mathrm{CDCl}_{3}\right): \delta 138.1$, 128.2, 55.3, 30.7, 18.6. HRMS: [M] $]^{+}$calcd for $\mathrm{C}_{24} \mathrm{H}_{46} \mathrm{~N}_{4}, 390.3722$; Found, 390.3719 .

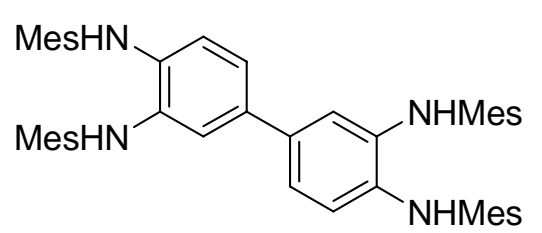

3,3',4,4'-Tetrakis(2,4,6-trimethylphenylamino)biphenyl (13). A catalyst for mediating aryl-amination coupling reactions was prepared by charging a $10 \mathrm{~mL}$ vial with $1,3-b i s(2,6-$ diisopropylphenyl)imidazolium chloride $(86 \mathrm{mg}, 0.02 \mathrm{mmol}$ ), $\mathrm{NaOtBu}(3 \mathrm{mg}, 0.03 \mathrm{mmol}), \mathrm{Pd}(\mathrm{OAc})_{2}(30 \mathrm{mg}, 0.01 \mathrm{mmol})$, toluene $(3 \mathrm{~mL})$, and a stir bar followed by stirring this mixture at $23{ }^{\circ} \mathrm{C}$ for 10 minutes. 2Bromomesitylene $(410 \mathrm{mg}, 2.05 \mathrm{mmol})$ was added to the catalyst solution. 3,3'diaminobenzidine $(110 \mathrm{mg}, 0.5 \mathrm{mmol})$ and $\mathrm{NaOtBu}(0.20 \mathrm{~g}, 2.05 \mathrm{mmol})$ were then added and the resulting mixture was sealed and stirred at $110{ }^{\circ} \mathrm{C}$ overnight. After cooling to ambient temperature, precipitated solids $(\mathrm{NaBr})$ were removed by filtration under a cone of nitrogen. Solvent was removed under reduced pressure. Analysis of the crude product indicated that the 
reaction was $>98 \%$ complete. The crude product was triturated with hexanes and then dried under reduced pressure to afford $160 \mathrm{mg}$ (47\% yield) of the desired product as a grey solid. ${ }^{1} \mathrm{H}$ NMR $\left(\mathrm{CDCl}_{3}\right): \delta 6.94(\mathrm{~s}, 8 \mathrm{H}), 6.68-6.66(\mathrm{~m}, 2 \mathrm{H}), 6.27-6.24(\mathrm{~m}, 4 \mathrm{H}), 5.18(\mathrm{br}, 2 \mathrm{H}), 4.99(\mathrm{br}$, 2H), $2.36(\mathrm{br}, 6 \mathrm{H}), 2.31(\mathrm{~s}, 6 \mathrm{H}), 2.17(\mathrm{~s}, 12 \mathrm{H}), 2.13(\mathrm{~s}, 12 \mathrm{H}) .{ }^{13} \mathrm{C} \mathrm{NMR}\left(\mathrm{CDCl}_{3}\right): \delta 137.11$, $137.06,136.7,135.7,135.6,134.0,133.9,133.54,133.48,133.3,133.2,133.1,129.30,129.26$, 117.8, 114.7, 112.4, 20.9, 20.8, 18.1, 18.0. HRMS: [M] ${ }^{+}$calcd for $\mathrm{C}_{48} \mathrm{H}_{55} \mathrm{~N}_{4}, 687.4427$; Found: 687.4423.

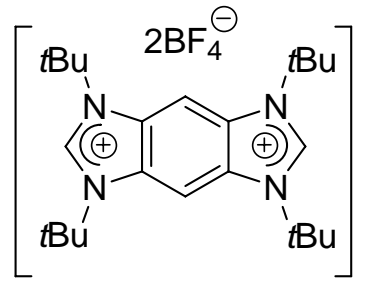

Tetrakis(tert-butyl) benzobis(imidazolium) tetrafluoroborate (14). A $10 \mathrm{~mL}$ flask was charged with triethylorthoformate $(5 \mathrm{~mL})$, compound 4 (590 mg, $1.63 \mathrm{mmol}$ ), and $\mathrm{HBF}_{4}$ (1 mL, 48\% aq.). The reaction mixture was heated to $65^{\circ} \mathrm{C}$ for $24 \mathrm{~h}$. Upon completion, the product was collected by filtration, washed with hot acetonitrile $(3 \times 15 \mathrm{~mL})$ to obtain $700 \mathrm{mg}$ $(77 \%)$ of the desired product as a light yellow powder. ${ }^{1} \mathrm{H}$ NMR (DMSO$\left.d_{6}\right): \delta 9.06(\mathrm{~s}, 2 \mathrm{H}), 8.62(\mathrm{~s}, 2 \mathrm{H}), 1.91(\mathrm{~s}, 36 \mathrm{H}) .{ }^{13} \mathrm{C}$ NMR $\left(\mathrm{DMSO}-d_{6}\right): \delta 142.9,128.9,102.7$, $62.0,28.0,9.1$.

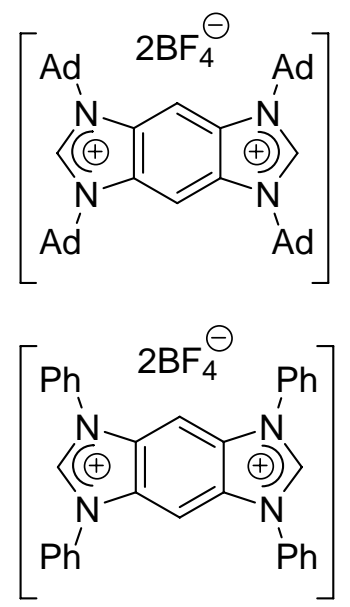

Tetraadamantyl benzobis(imidazolium) tetrafluoroborate (15). In a manner analogous to 14 using tetraamine 6 (3.3 g, $4.89 \mathrm{mmol})$, the product was obtained in $70 \%$ yield as a light yellow powder. ${ }^{1} \mathrm{H}$ NMR $\left(\mathrm{C}_{6} \mathrm{D}_{6}\right): \delta$ $9.13(\mathrm{~s}, 2 \mathrm{H}), 8.54(\mathrm{~s}, 2 \mathrm{H}), 2.39(\mathrm{~s}, 12 \mathrm{H}), 1.88-1.76$ (br, 24H). ${ }^{13} \mathrm{C} \mathrm{NMR}$ $\left(\mathrm{C}_{6} \mathrm{D}_{6}\right): \delta 142.9,127.9,103.3,62.7,40.4,35.4,29.1$.

\section{Tetraphenyl benzobis(imidazolium) tetrafluoroborate (16).}

In a manner analogous to 14 using tetraamine $7(0.3 \mathrm{~g}, 0.68 \mathrm{mmol})$, with a reaction temperature of $140{ }^{\circ} \mathrm{C}$ and time of $24 \mathrm{~h} 162 \mathrm{mg}(51 \%)$ of the desired product was obtained as a light yellow powder (purification required rinsing with copious amounts of $\mathrm{MeOH}) .{ }^{1} \mathrm{H}$ NMR (DMSO- $\left.d_{6}\right) \delta$ $10.8(\mathrm{~s}, 2 \mathrm{H}), 8.15(\mathrm{~s}, 2 \mathrm{H}) 7.9-7.8(\mathrm{br}, 20 \mathrm{H}) .{ }^{13} \mathrm{C}$ NMR $\left(\mathrm{DMSO}-d_{6}\right) \delta 147.3$, 132.9, 131.0, 130.6, 125.6, 99.2. HRMS: [M] ${ }^{2+}$ calcd for $\mathrm{C}_{32} \mathrm{H}_{24} \mathrm{~N}_{4}, 232.1000$; Found: 232.1002 . Alternatively, a one-pot, two-step synthesis of 16 may be accomplished in $84 \%$ overall yield by eliminating the workup of the tetrakis(phenylamino)benzene. Aryl amination reaction of 1,2,4,5tetrabromobenzene (6 $\mathrm{g}, 15.2 \mathrm{mmol})$ was carried out as described above. After cooling the reaction vessel, the reaction mixture was acidified with $\mathrm{HBF}_{4}(5 \mathrm{~mL}, 48 \%$ aq. $)$ and $\mathrm{HC}(\mathrm{OEt})_{3}$ (200 mL) was added. The vessel was then heated to $140{ }^{\circ} \mathrm{C}$ for $16 \mathrm{~h}$, then cooled to $23{ }^{\circ} \mathrm{C}$. Additional $\mathrm{HBF}_{4}(3 \mathrm{~mL})$ was added and the solution was re-heated to $140{ }^{\circ} \mathrm{C}$ for $6 \mathrm{~h}$. After cooling to room temperature, the resulting precipitate was collected by filtration and then washed with water $(3 \times 50 \mathrm{~mL})$, methanol $(1 \times 50 \mathrm{~mL})$, and ether $(2 \times 30 \mathrm{~mL})$ to obtain $8.2 \mathrm{~g}$ (84\% yield) of analytically pure product as a brown solid.

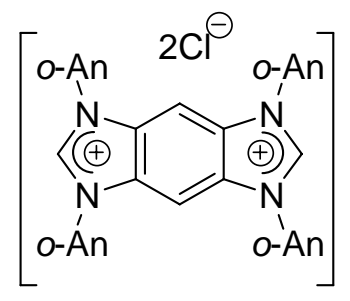

Tetrakis(2-methoxyphenyl) benzobis(imidazolium) chloride (17). The tetrahydrochloride of tetraamine $10(5.0 \mathrm{~g}, 7.06 \mathrm{mmol})$ was dissolved in $\mathrm{HC}(\mathrm{OEt})_{3}(60 \mathrm{~mL})$ and conc. $\mathrm{HCl}(0.2 \mathrm{~mL})$. The deep purple solution was stirred at $120{ }^{\circ} \mathrm{C}$ for $2 \mathrm{~h}$ during which time the homogenous reaction mixture became a light red slurry. The reaction mixture was allowed to cool and $\mathrm{Et}_{2} \mathrm{O}(30 \mathrm{~mL})$ was added to facilitate precipitation of the product. The 
solids were collected, rinsed with $\mathrm{Et}_{2} \mathrm{O}$ and recrystallized from hot $\mathrm{MeOH} / \mathrm{Et}_{2} \mathrm{O}$ to give $4.26 \mathrm{~g}$ $(92 \%)$ of the bis(azolium) as a pink-brown solid. ${ }^{1} \mathrm{H}$ NMR (DMSO- $\left.d_{6}\right): \delta 10.79(\mathrm{~s}, 2 \mathrm{H}), 8.00$ (dd, $J=7.8,1.4 \mathrm{~Hz}, 2 \mathrm{H}), 7.98(\mathrm{~s}, 2 \mathrm{H}), 7.73(\mathrm{t}, J=8.0 \mathrm{~Hz}, 4 \mathrm{H}), 7.48(\mathrm{~d}, J=6.8 \mathrm{~Hz}, 4 \mathrm{H}), 7.28(\mathrm{t}, J=$ $7.2 \mathrm{~Hz}, 4 \mathrm{H}), 3.87(\mathrm{~s}, 12 \mathrm{H}) .{ }^{13} \mathrm{C}$ NMR (DMSO-d $): \delta 153.7,148.5,133.0,130.8,128.4,121.3$, 120.6, 113.7, 100.4, 56.4. HRMS: [M] ${ }^{+}$calcd for $\mathrm{C}_{36} \mathrm{H}_{32} \mathrm{~N}_{4} \mathrm{O}_{4}, 584.2462$; Found: 584.2418. 
X-ray Crystallographic Data for 4:<smiles>CCCCNc1cc(NCCCC)c(NCC(C)(C)C)cc1NCCCC</smiles>

Figure 1. View of 1 showing the atom labeling scheme. Displacement ellipsoids are scaled to the $50 \%$ probability level. The macrocycle lies on a crystallographic inversion center at $1 / 2,1 / 2,1 / 2$. Atoms with labels appended by a ' are related by 1-x, 1-y, 1-z.

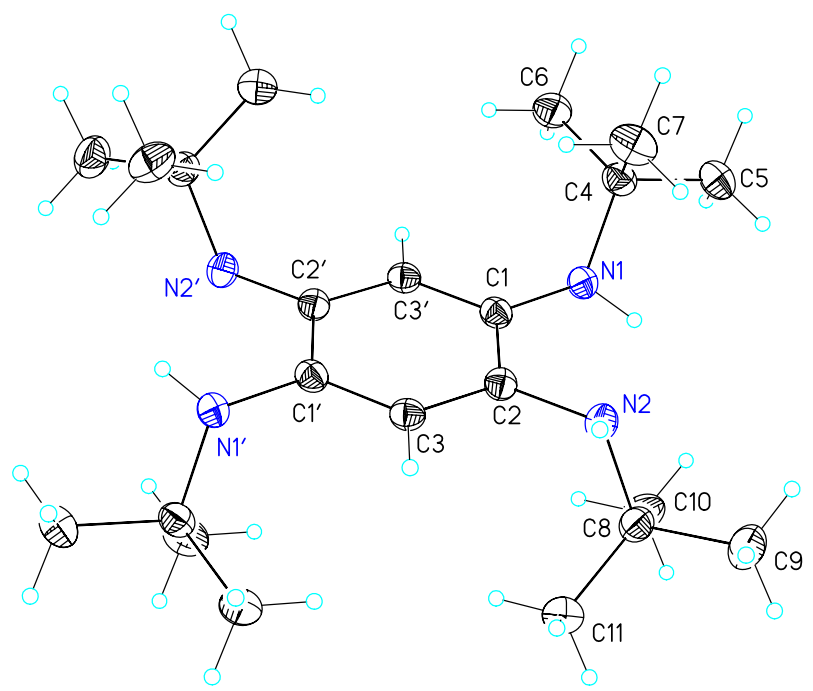

X-ray Experimental for $4\left(\mathrm{C}_{22} \mathrm{H}_{42} \mathrm{~N}_{4}\right)$ : Crystals grew as large, colorless needles by slow evaporation from toluene. The data crystal was cut from a larger crystal and had approximate dimensions; $0.34 \times 0.28 \times 0.24 \mathrm{~mm}$. The data were collected on a Nonius Kappa CCD diffractometer using a graphite monochromator with $\mathrm{MoK} \alpha$ radiation $(\lambda=0.71073 \AA)$. A total of 417 frames of data were collected using $\omega$-scans with a scan range of $1^{\circ}$ and a counting time of 101 seconds per frame. The data were collected at $153 \mathrm{~K}$ using an Oxford Cryostream low temperature device. Details of crystal data, data collection and structure refinement are listed in Table 1. Data reduction were performed using DENZO-SMN. ${ }^{1}$ The structure was solved by direct methods using SIR $97^{2}$ and refined by full-matrix least-squares on $\mathrm{F}^{2}$ with anisotropic displacement parameters for the non-H atoms using SHELXL-97. ${ }^{3}$ The methyl group hydrogen atoms were calculated in ideal positions with isotropic displacement parameters set to $1.5 \mathrm{xUeq}$ 
of the attached atom. The hydrogen atoms on nitrogen and the ring carbon atoms were observed in a $\Delta \mathrm{F}$ map and refined with isotropic displacement parameters. The function, $\Sigma \mathrm{w}\left(\left|\mathrm{F}_{\mathrm{o}}\right|^{2}-\left|\mathrm{F}_{\mathrm{c}}\right|^{2}\right)^{2}$, was minimized, where $\mathrm{w}=1 /\left[\left(\sigma\left(\mathrm{F}_{\mathrm{o}}\right)\right)^{2}+(0.0591 * \mathrm{P})^{2}+(0.1657 * \mathrm{P})\right]$ and $\mathrm{P}=\left(\left|\mathrm{F}_{\mathrm{o}}\right|^{2}+2\left|\mathrm{~F}_{\mathrm{c}}\right|^{2}\right) / 3$. $\mathrm{R}_{\mathrm{W}}\left(\mathrm{F}^{2}\right)$ refined to 0.129 , with $\mathrm{R}(\mathrm{F})$ equal to 0.0462 and a goodness of fit, $\mathrm{S},=1.02$. Definitions used for calculating $\mathrm{R}(\mathrm{F}), \mathrm{R}_{\mathrm{W}}\left(\mathrm{F}^{2}\right)$ and the goodness of fit, $\mathrm{S}$, are given below. ${ }^{4}$ The data were corrected for secondary extinction effects. The correction takes the form: $\mathrm{F}_{\mathrm{corr}}=\mathrm{kF}_{\mathrm{c}} /[1+$ $\left.\left(3.7(13) \times 10^{-5}\right)^{*} \mathrm{~F}_{\mathrm{c}}^{2} \lambda^{3 /(\sin 2 \theta)}\right]^{0.25}$ where $\mathrm{k}$ is the overall scale factor. Neutral atom scattering factors and values used to calculate the linear absorption coefficient are from the International Tables for X-ray Crystallography (1992). ${ }^{5}$ All figures were generated using SHELXTL/PC. 6 Tables of positional and thermal parameters, bond lengths and angles, torsion angles, figures and lists of observed and calculated structure factors are located in tables 1 through 7.

\section{$\underline{\text { References }}$}

1) DENZO-SMN. (1997). Z. Otwinowski and W. Minor, Methods in Enzymology, 276: Macromolecular Crystallography, part A, 307 - 326, C. W. Carter, Jr. and R. M. Sweets, Editors, Academic Press.

2) SIR97. (1999). A program for crystal structure solution. Altomare A., Burla M.C., Camalli M., Cascarano G.L., Giacovazzo C. , Guagliardi A., Moliterni A.G.G., Polidori G.,Spagna R. J. Appl. Cryst. 32, 115-119.

3) Sheldrick, G. M. (1994). SHELXL97. Program for the Refinement of Crystal Structures. University of Gottingen, Germany.

4) $\quad \mathrm{R}_{\mathrm{W}}\left(\mathrm{F}^{2}\right)=\left\{\sum \mathrm{w}\left(\left|\mathrm{F}_{\mathrm{O}}\right|^{2}-\left|\mathrm{F}_{\mathrm{c}}\right|^{2}\right)^{2 / \Sigma \mathrm{w}}\left(\left|\mathrm{F}_{\mathrm{O}}\right|\right)^{4}\right\}^{1 / 2}$ where $\mathrm{w}$ is the weight given each reflection. $\left.\mathrm{R}(\mathrm{F})=\Sigma\left(\left|\mathrm{F}_{\mathrm{O}}\right|-\left|\mathrm{F}_{\mathrm{C}}\right|\right) / \Sigma\left|\mathrm{F}_{\mathrm{O}}\right|\right\}$ for reflections with $\mathrm{F}_{\mathrm{O}}>4\left(\sigma\left(\mathrm{F}_{\mathrm{O}}\right)\right)$. $\mathrm{S}=\left[\sum \mathrm{w}\left(\left|\mathrm{F}_{\mathrm{O}}\right|^{2}-\left|\mathrm{F}_{\mathrm{C}}\right|^{2}\right)^{2 /(n-p)}\right]^{1 / 2}$, where $\mathrm{n}$ is the number of reflections and $\mathrm{p}$ is the number of refined parameters.

5) International Tables for X-ray Crystallography (1992). Vol. C, Tables 4.2.6.8 and 6.1.1.4, A. J. C. Wilson, editor, Boston: Kluwer Academic Press.

6) Sheldrick, G. M. (1994). SHELXTL/PC (Version 5.03). Siemens Analytical Xray Instruments, Inc., Madison, Wisconsin, USA. 
Table 1. Crystal data and structure refinement for 4.

Empirical formula

Formula weight

Temperature

Wavelength

Crystal system

Space group

Unit cell dimensions

Volume

Z

Density (calculated)

Absorption coefficient

$\mathrm{F}(000)$

Crystal size

Theta range for data collection

Index ranges

Reflections collected

Independent reflections

Completeness to theta $=27.50^{\circ}$

Absorption correction

Refinement method

Data / restraints / parameters

Goodness-of-fit on $\mathrm{F}^{2}$

Final $\mathrm{R}$ indices $[\mathrm{I}>2 \operatorname{sigma}(\mathrm{I})]$

$\mathrm{R}$ indices (all data)

Extinction coefficient

Largest diff. peak and hole
C22 H42 N4

362.60

153(2) K

$0.71073 \AA$

Triclinic

P-1

$\mathrm{a}=5.9656(2) \AA \quad \alpha=71.1320(10)^{\circ}$.

$\mathrm{b}=8.8102(2) \AA \quad \beta=78.6270(10)^{\circ}$.

$\mathrm{c}=11.4701(4) \AA \quad \gamma=86.5320(10)^{\circ}$.

$559.25(3) \AA^{3}$

1

$1.077 \mathrm{Mg} / \mathrm{m}^{3}$

$0.064 \mathrm{~mm}^{-1}$

202

$0.34 \times 0.28 \times 0.24 \mathrm{~mm}$

3.48 to $27.50^{\circ}$.

$-6<=\mathrm{h}<=7,-9<=\mathrm{k}<=11,-10<=\mathrm{l}<=14$

3890

$2558[\mathrm{R}(\mathrm{int})=0.0175]$

$99.1 \%$

None

Full-matrix least-squares on $\mathrm{F}^{2}$

$2558 / 0 / 131$

1.017

$\mathrm{R} 1=0.0462, \mathrm{wR} 2=0.1135$

$\mathrm{R} 1=0.0713, \mathrm{wR} 2=0.1291$

$3.7(13) \times 10^{-5}$

0.249 and -0.184 e. $\AA^{-3}$ 
Table 2. Atomic coordinates ( x 104) and equivalent isotropic displacement parameters $\left(\AA^{2} \mathrm{x}\right.$ $10^{3}$ ) for 4 . $U(e q)$ is defined as one third of the trace of the orthogonalized $U^{i j}$ tensor.

\begin{tabular}{lcccc}
\hline & $\mathrm{x}$ & $\mathrm{y}$ & $\mathrm{z}$ & $\mathrm{U}(\mathrm{eq})$ \\
\hline $\mathrm{N} 1$ & & & & \\
$\mathrm{~N} 2$ & $1658(2)$ & $4451(1)$ & $7241(1)$ & $21(1)$ \\
$\mathrm{C} 1$ & $6049(2)$ & $5731(1)$ & $7076(1)$ & $20(1)$ \\
$\mathrm{C} 2$ & $3351(2)$ & $4723(2)$ & $6122(1)$ & $19(1)$ \\
$\mathrm{C} 3$ & $5493(2)$ & $5403(2)$ & $6014(1)$ & $18(1)$ \\
$\mathrm{C} 4$ & $7094(2)$ & $5639(2)$ & $4907(1)$ & $19(1)$ \\
C5 & $1117(2)$ & $2759(2)$ & $8062(1)$ & $23(1)$ \\
C6 & $-367(3)$ & $2903(2)$ & $9266(1)$ & $31(1)$ \\
C7 & $-237(2)$ & $1895(2)$ & $7465(1)$ & $29(1)$ \\
C8 & $3293(3)$ & $1822(2)$ & $8354(2)$ & $33(1)$ \\
C9 & $5805(2)$ & $7414(2)$ & $7118(1)$ & $23(1)$ \\
C10 & $6563(3)$ & $7371(2)$ & $8321(1)$ & $31(1)$ \\
C11 & $3318(2)$ & $7928(2)$ & $7168(2)$ & $31(1)$ \\
& $7293(3)$ & $8610(2)$ & $5991(1)$ & $31(1)$ \\
\hline
\end{tabular}


Table 3. Bond lengths $[\AA]$ and angles $\left[{ }^{\circ}\right]$ for 4.

\begin{tabular}{|c|c|c|c|}
\hline $\mathrm{N} 1-\mathrm{C} 1$ & $1.4294(17)$ & C6-H6B & 0.96 \\
\hline $\mathrm{N} 1-\mathrm{C} 4$ & $1.4980(17)$ & C6-H6C & 0.96 \\
\hline N1-H1N & $0.908(18)$ & C7-H7A & 0.96 \\
\hline N2-C2 & $1.4406(17)$ & C7-H7B & 0.96 \\
\hline N2-C8 & $1.4967(18)$ & C7-H7C & 0.96 \\
\hline $\mathrm{N} 2-\mathrm{H} 2 \mathrm{~N}$ & $0.912(17)$ & $\mathrm{C} 8-\mathrm{C} 10$ & $1.5201(19$ \\
\hline C1-C3\#1 & $1.3938(19)$ & C8-C9 & $1.524(2)$ \\
\hline $\mathrm{C} 1-\mathrm{C} 2$ & $1.4107(18)$ & C8-C11 & $1.533(2)$ \\
\hline $\mathrm{C} 2-\mathrm{C} 3$ & $1.3941(19)$ & C9-H9A & 0.96 \\
\hline $\mathrm{C} 3-\mathrm{C} 1 \# 1$ & $1.3938(19)$ & C9-H9B & 0.96 \\
\hline C3-H3 & $0.982(16)$ & C9-H9C & 0.96 \\
\hline $\mathrm{C} 4-\mathrm{C} 5$ & $1.525(2)$ & C10-H10A & 0.96 \\
\hline C4-C6 & $1.5264(19)$ & C10-H10B & 0.96 \\
\hline $\mathrm{C} 4-\mathrm{C} 7$ & $1.5299(19)$ & C10-H10C & 0.96 \\
\hline C5-H5A & 0.96 & C11-H11A & 0.96 \\
\hline C5-H5B & 0.96 & C11-H11B & 0.96 \\
\hline C5-H5C & 0.96 & C11-H11C & 0.96 \\
\hline C6-H6A & 0.96 & & \\
\hline C1-N1-C4 & $118.63(11)$ & N1-C4-C5 & $105.06(11)$ \\
\hline C1-N1-H1N & $107.7(11)$ & N1-C4-C6 & $111.31(11)$ \\
\hline C4-N1-H1N & $103.7(11)$ & $\mathrm{C} 5-\mathrm{C} 4-\mathrm{C} 6$ & $109.10(12)$ \\
\hline C2-N2-C8 & $118.30(11)$ & N1-C4-C7 & $111.31(11)$ \\
\hline $\mathrm{C} 2-\mathrm{N} 2-\mathrm{H} 2 \mathrm{~N}$ & $106.0(10)$ & $\mathrm{C} 5-\mathrm{C} 4-\mathrm{C} 7$ & $109.97(12)$ \\
\hline $\mathrm{C} 8-\mathrm{N} 2-\mathrm{H} 2 \mathrm{~N}$ & $106.8(10)$ & C6-C4-C7 & $109.97(12)$ \\
\hline $\mathrm{C} 3 \# 1-\mathrm{C} 1-\mathrm{C} 2$ & $118.06(12)$ & C4-C5-H5A & 109.5 \\
\hline C3\#1-C1-N1 & $120.82(12)$ & C4-C5-H5B & 109.5 \\
\hline $\mathrm{C} 2-\mathrm{C} 1-\mathrm{N} 1$ & $121.10(12)$ & H5A-C5-H5B & 109.5 \\
\hline $\mathrm{C} 3-\mathrm{C} 2-\mathrm{C} 1$ & $119.06(12)$ & $\mathrm{C} 4-\mathrm{C} 5-\mathrm{H} 5 \mathrm{C}$ & 109.5 \\
\hline $\mathrm{C} 3-\mathrm{C} 2-\mathrm{N} 2$ & $121.18(11)$ & $\mathrm{H} 5 \mathrm{~A}-\mathrm{C} 5-\mathrm{H} 5 \mathrm{C}$ & 109.5 \\
\hline $\mathrm{C} 1-\mathrm{C} 2-\mathrm{N} 2$ & $119.60(12)$ & H5B-C5-H5C & 109.5 \\
\hline $\mathrm{C} 1 \# 1-\mathrm{C} 3-\mathrm{C} 2$ & $122.85(12)$ & C4-C6-H6A & 109.2 \\
\hline $\mathrm{C} 1 \# 1-\mathrm{C} 3-\mathrm{H} 3$ & 119.2(9) & C4-C6-H6B & 109.5 \\
\hline C2-C3-H3 & $118.0(9)$ & H6A-C6-H6B & 109.5 \\
\hline
\end{tabular}




$\begin{array}{ll}\text { C4-C6-H6C } & 109.8 \\ \text { H6A-C6-H6C } & 109.5 \\ \text { H6B-C6-H6C } & 109.5 \\ \text { C4-C7-H7A } & 109.7 \\ \text { C4-C7-H7B } & 109.6 \\ \text { H7A-C7-H7B } & 109.5 \\ \text { C4-C7-H7C } & 109.2 \\ \text { H7A-C7-H7C } & 109.5 \\ \text { H7B-C7-H7C } & 109.5 \\ \text { N2-C8-C10 } & 109.84(11) \\ \text { N2-C8-C9 } & 105.53(11) \\ \text { C10-C8-C9 } & 109.94(12) \\ \text { N2-C8-C11 } & 112.64(12) \\ \text { C10-C8-C11 } & 109.20(12) \\ \text { C9-C8-C11 } & 109.63(12) \\ \text { C8-C9-H9A } & 108.9 \\ \text { C8-C9-H9B } & 109.3 \\ \text { H9A-C9-H9B } & 109.5 \\ \text { C8-C9-H9C } & 110.2 \\ \text { H9A-C9-H9C } & 109.5 \\ \text { H9B-C9-H9C } & 109.5 \\ \text { C8-C10-H10A } & 109.9 \\ \text { C8-C10-H10B } & 110.2 \\ \text { H10A-C10-H10B } & 109.5 \\ \text { C8-C10-H10C } & 108.3 \\ \text { H10A-C10-H10C } & 109.5 \\ \text { H10B-C10-H10C } & 109.5 \\ \text { C8-C11-H11A } & 109.7 \\ \text { C8-C11-H11B } & 108.9 \\ \text { H11A-C11-H11B } & 109.5 \\ \text { C8-C11-H11C } & 109.8 \\ \text { H11A-C11-H11C } & 109.5 \\ \text { H11B-C11-H11C } & 109.5 \\ & \end{array}$


Symmetry transformations used to generate equivalent atoms:

$\# 1-\mathrm{x}+1,-\mathrm{y}+1,-\mathrm{z}+1$ 
Table 4. Anisotropic displacement parameters $\left(\AA^{2} \times 10^{3}\right)$ for 4. The anisotropic displacement factor exponent takes the form: $-2 \pi^{2}\left[\mathrm{~h}^{2} \mathrm{a}^{* 2} \mathrm{U}^{11}+\ldots+2 \mathrm{hk} \mathrm{a}^{*} \mathrm{~b}^{*} \mathrm{U}^{12}\right]$

\begin{tabular}{lllllll}
\hline & $\mathrm{U} 11$ & $\mathrm{U}^{22}$ & $\mathrm{U}^{33}$ & $\mathrm{U}^{23}$ & $\mathrm{U} 13$ & $\mathrm{U}^{12}$ \\
\hline $\mathrm{N} 1$ & $22(1)$ & $22(1)$ & $19(1)$ & $-7(1)$ & $1(1)$ & $-3(1)$ \\
$\mathrm{N} 2$ & $20(1)$ & $23(1)$ & $20(1)$ & $-8(1)$ & $-5(1)$ & $1(1)$ \\
$\mathrm{C} 1$ & $18(1)$ & $18(1)$ & $19(1)$ & $-5(1)$ & $-2(1)$ & $1(1)$ \\
$\mathrm{C} 2$ & $19(1)$ & $18(1)$ & $18(1)$ & $-5(1)$ & $-5(1)$ & $2(1)$ \\
$\mathrm{C} 3$ & $17(1)$ & $19(1)$ & $21(1)$ & $-6(1)$ & $-3(1)$ & $-2(1)$ \\
$\mathrm{C} 4$ & $22(1)$ & $22(1)$ & $22(1)$ & $-5(1)$ & $-1(1)$ & $-3(1)$ \\
$\mathrm{C} 5$ & $33(1)$ & $32(1)$ & $23(1)$ & $-5(1)$ & $2(1)$ & $-6(1)$ \\
$\mathrm{C} 6$ & $27(1)$ & $27(1)$ & $30(1)$ & $-8(1)$ & $-1(1)$ & $-7(1)$ \\
$\mathrm{C} 7$ & $29(1)$ & $29(1)$ & $35(1)$ & $-3(1)$ & $-6(1)$ & $0(1)$ \\
$\mathrm{C} 8$ & $22(1)$ & $24(1)$ & $23(1)$ & $-10(1)$ & $-4(1)$ & $-2(1)$ \\
$\mathrm{C} 9$ & $34(1)$ & $37(1)$ & $29(1)$ & $-17(1)$ & $-7(1)$ & $-3(1)$ \\
$\mathrm{C} 10$ & $27(1)$ & $28(1)$ & $42(1)$ & $-18(1)$ & $-7(1)$ & $3(1)$ \\
$\mathrm{C} 11$ & $36(1)$ & $26(1)$ & $29(1)$ & $-8(1)$ & $-3(1)$ & $-6(1)$ \\
& & & & & & \\
\hline
\end{tabular}


Table 5. Hydrogen coordinates ( x 104) and isotropic displacement parameters $\left(\AA^{2} \times 10^{3}\right)$ for 4 .

\begin{tabular}{|c|c|c|c|c|}
\hline & $\mathrm{x}$ & $\mathrm{y}$ & $\mathrm{z}$ & $\mathrm{U}(\mathrm{eq})$ \\
\hline H5A & 480 & 3440 & 9649 & 47 \\
\hline H5B & -1714 & 3509 & 9073 & 47 \\
\hline $\mathrm{H} 5 \mathrm{C}$ & -800 & 1852 & 9835 & 47 \\
\hline H6A & -1599 & 2491 & 7290 & 43 \\
\hline H6B & 678 & 1819 & 6698 & 43 \\
\hline $\mathrm{H} 6 \mathrm{C}$ & -645 & 837 & 8030 & 43 \\
\hline H7A & 4137 & 2367 & 8731 & 49 \\
\hline H7B & 2893 & 764 & 8921 & 49 \\
\hline $\mathrm{H} 7 \mathrm{C}$ & 4216 & 1745 & 7589 & 49 \\
\hline H9A & 5619 & 6620 & 9020 & 47 \\
\hline H9B & 8129 & 7036 & 8284 & 47 \\
\hline H9C & 6414 & 8415 & 8422 & 47 \\
\hline $\mathrm{H} 10 \mathrm{~A}$ & 2371 & 7192 & 7876 & 46 \\
\hline H10B & 3139 & 8989 & 7235 & 46 \\
\hline $\mathrm{H} 10 \mathrm{C}$ & 2874 & 7923 & 6409 & 46 \\
\hline H11A & 8871 & 8306 & 5962 & 46 \\
\hline H11B & 6834 & 8602 & 5236 & 46 \\
\hline $\mathrm{H} 11 \mathrm{C}$ & 7099 & 9668 & 6062 & 46 \\
\hline $\mathrm{H} 2 \mathrm{~N}$ & $7550(30)$ & $5463(19)$ & $7076(15)$ & $28(4)$ \\
\hline $\mathrm{H} 1 \mathrm{~N}$ & $2170(30)$ & $4900(20)$ & $7747(16)$ & $32(4)$ \\
\hline H3 & $8600(30)$ & $6080(19)$ & $4858(14)$ & $28(4)$ \\
\hline
\end{tabular}


Table 6. Torsion angles $\left[^{\circ}\right]$ for 4.

\begin{tabular}{lc}
\hline C4-N1-C1-C3\#1 & $71.81(16)$ \\
C4-N1-C1-C2 & $-109.84(14)$ \\
C3\#1-C1-C2-C3 & $-1.9(2)$ \\
N1-C1-C2-C3 & $179.72(11)$ \\
C3\#1-C1-C2-N2 & $-177.41(11)$ \\
N1-C1-C2-N2 & $4.20(19)$ \\
C8-N2-C2-C3 & $84.72(16)$ \\
C8-N2-C2-C1 & $-99.85(15)$ \\
C1-C2-C3-C1\#1 & $2.0(2)$ \\
N2-C2-C3-C1\#1 & $177.43(12)$ \\
C1-N1-C4-C5 & $169.45(12)$ \\
C1-N1-C4-C6 & $-72.60(15)$ \\
C1-N1-C4-C7 & $50.47(16)$ \\
C2-N2-C8-C10 & $62.78(15)$ \\
C2-N2-C8-C9 & $-178.76(11)$ \\
C2-N2-C8-C11 & $-59.18(15)$ \\
\end{tabular}

Symmetry transformations used to generate equivalent atoms:

\#1 - $\mathrm{x}+1,-\mathrm{y}+1,-\mathrm{z}+1$ 
X-ray Crystallographic Data for 5:<smiles>CCCCNC1=CC(=NCC(C)C)C(NCC(C)(C)C)=CC1=NCC(C)C</smiles>

Figure 2. View of 5 showing the atom labeling scheme. Displacement ellipsoids are scaled to the $50 \%$ probability level. The molecule lies around a crystallographic inversion center at $1 / 2,1 / 2$, $1 / 2$. Atoms with labels appended by a ' are related by 1-x, 1-y, 1-z.

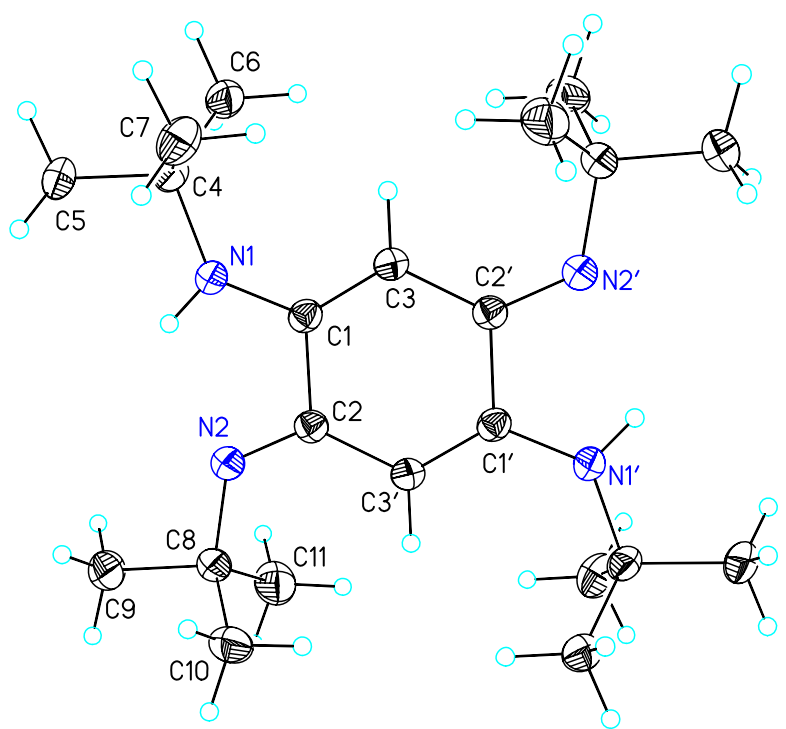

$\mathrm{X}$-ray Experimental for $5\left(\mathrm{C}_{22} \mathrm{H}_{40} \mathrm{~N}_{4}\right)$ : Crystals grew as large orange prisms by slow evaporation from chloroform. The data crystal was cut from a much larger crystal and had approximate dimensions; $0.28 \times 0.25 \times 0.18 \mathrm{~mm}$. The data were collected on a Nonius Kappa CCD diffractometer using a graphite monochromator with MoK $\alpha$ radiation $(\lambda=0.71073 \AA)$. A total of 259 frames of data were collected using $\omega$-scans with a scan range of $2^{\circ}$ and a counting time of 104 seconds per frame. The data were collected at $153 \mathrm{~K}$ using an Oxford Cryostream low temperature device. Details of crystal data, data collection and structure refinement are listed in Table 1. Data reduction were performed using DENZO-SMN. ${ }^{1}$ The structure was solved by direct methods using SIR97 ${ }^{2}$ and refined by full-matrix least-squares on $\mathrm{F}^{2}$ with anisotropic displacement parameters for the non-H atoms using SHELXL-97. ${ }^{3}$ The hydrogen atoms on carbon were observed in a $\Delta \mathrm{F}$ map and refined with isotropic displacement parameters. The molecule resides around a crystallographic inversion center at $1 / 2,1 / 2,1 / 2$. The function, $\Sigma w\left(\left|\mathrm{~F}_{\mathrm{O}}\right|^{2}-\right.$ $\left.\left|\mathrm{F}_{\mathrm{c}}\right|^{2}\right)^{2}$, was minimized, where $\mathrm{w}=1 /\left[\left(\sigma\left(\mathrm{F}_{\mathrm{o}}\right)\right)^{2}+\left(0.0433^{*} \mathrm{P}\right)^{2}+(0.2874 * \mathrm{P})\right]$ and $\mathrm{P}=\left(\left|\mathrm{F}_{\mathrm{o}}\right|^{2}+\right.$ $\left.2\left|\mathrm{~F}_{\mathrm{c}}\right|^{2}\right) / 3 . \quad \mathrm{R}_{\mathrm{w}}\left(\mathrm{F}^{2}\right)$ refined to 0.0957 , with $\mathrm{R}(\mathrm{F})$ equal to 0.0370 and a goodness of fit, $\mathrm{S},=1.01$. Definitions used for calculating $\mathrm{R}(\mathrm{F}), \mathrm{R}_{\mathrm{w}}\left(\mathrm{F}^{2}\right)$ and the goodness of fit, $\mathrm{S}$, are given below. ${ }^{4}$ The data were checked for secondary extinction effects but no correction was necessary. Neutral 
atom scattering factors and values used to calculate the linear absorption coefficient are from the International Tables for X-ray Crystallography (1992). ${ }^{5}$ All figures were generated using SHELXTL/PC. ${ }^{6}$ Tables of positional and thermal parameters, bond lengths and angles, torsion angles, figures and lists of observed and calculated structure factors are located in tables 1 through

\section{References}

6) DENZO-SMN. (1997). Z. Otwinowski and W. Minor, Methods in Enzymology, 276: Macromolecular Crystallography, part A, 307 - 326, C. W. Carter, Jr. and R. M. Sweets, Editors, Academic Press.

7) SIR97. (1999). A program for crystal structure solution. Altomare A., Burla M.C., Camalli M., Cascarano G.L., Giacovazzo C. , Guagliardi A., Moliterni A.G.G., Polidori G.,Spagna R. J. Appl. Cryst. 32, 115-119.

8) Sheldrick, G. M. (1994). SHELXL97. Program for the Refinement of Crystal Structures. University of Gottingen, Germany.

9) $\quad \mathrm{R}_{\mathrm{W}}\left(\mathrm{F}^{2}\right)=\left\{\sum \mathrm{w}\left(\left|\mathrm{F}_{\mathrm{O}}\right|^{2}-\left|\mathrm{F}_{\mathrm{c}}\right|^{2}\right)^{2 / \Sigma \mathrm{w}}\left(\left|\mathrm{F}_{\mathrm{O}}\right|\right)^{4}\right\}^{1 / 2}$ where $\mathrm{w}$ is the weight given each reflection.

$\left.\mathrm{R}(\mathrm{F})=\Sigma\left(\left|\mathrm{F}_{\mathrm{O}}\right|-\left|\mathrm{F}_{\mathrm{c}}\right|\right) / \Sigma\left|\mathrm{F}_{\mathrm{O}}\right|\right\}$ for reflections with $\mathrm{F}_{\mathrm{O}}>4\left(\sigma\left(\mathrm{F}_{\mathrm{O}}\right)\right)$.

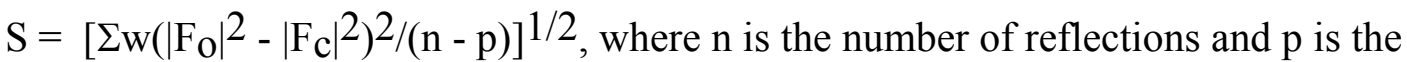
number of refined parameters.

10) International Tables for X-ray Crystallography (1992). Vol. C, Tables 4.2.6.8 and 6.1.1.4, A. J. C. Wilson, editor, Boston: Kluwer Academic Press.

6) Sheldrick, G. M. (1994). SHELXTL/PC (Version 5.03). Siemens Analytical Xray Instruments, Inc., Madison, Wisconsin, USA. 
Table 1. Crystal data and structure refinement for 5.

Empirical formula

Formula weight

Temperature

Wavelength

Crystal system

Space group

Unit cell dimensions

Volume

Z

Density (calculated)

Absorption coefficient

$\mathrm{F}(000)$

Crystal size

Theta range for data collection

Index ranges

Reflections collected

Independent reflections

Completeness to theta $=27.48^{\circ}$

Max. and min. transmission

Refinement method

Data / restraints / parameters

Goodness-of-fit on $\mathrm{F}^{2}$

Final R indices $[\mathrm{I}>2 \operatorname{sigma}(\mathrm{I})]$

$\mathrm{R}$ indices (all data)

Largest diff. peak and hole
C22 H40 N4

360.58

153(2) K

$0.71073 \AA$

Monoclinic

$\mathrm{P} 21 / \mathrm{c}$

$\mathrm{a}=10.6527(2) \AA$

$\alpha=90^{\circ}$.

$\mathrm{b}=10.3083(2) \AA$

$\beta=114.116(1)^{\circ}$.

$\mathrm{c}=11.3530(2) \AA$

$\gamma=90^{\circ}$.
2

$1.052 \mathrm{Mg} / \mathrm{m}^{3}$

$0.063 \mathrm{~mm}^{-1}$

400

$0.28 \times 0.25 \times 0.18 \mathrm{~mm}$

2.88 to $27.48^{\circ}$.

$-13<=\mathrm{h}<=13,-12<=\mathrm{k}<=13,-14<=\mathrm{l}<=14$

4951

$2600[\mathrm{R}(\mathrm{int})=0.0132]$

$99.7 \%$

0.9888 and 0.9827

Full-matrix least-squares on $\mathrm{F}^{2}$

2600 / 0 / 198

1.013

$\mathrm{R} 1=0.0370, \mathrm{wR} 2=0.0906$

$\mathrm{R} 1=0.0455, \mathrm{wR} 2=0.0957$

0.222 and -0.180 e. $\AA^{-3}$ 
Table 2. Atomic coordinates ( x 104) and equivalent isotropic displacement parameters $\left(\AA^{2} \mathrm{x}\right.$ $10^{3}$ ) for 5 . $U(e q)$ is defined as one third of the trace of the orthogonalized $U^{i j}$ tensor.

\begin{tabular}{lcccc}
\hline & $\mathrm{x}$ & $\mathrm{y}$ & $\mathrm{z}$ & $\mathrm{U}(\mathrm{eq})$ \\
\hline $\mathrm{N} 1$ & & & \\
$\mathrm{~N} 2$ & $4706(1)$ & $2500(1)$ & $5765(1)$ & $25(1)$ \\
$\mathrm{C} 1$ & $6982(1)$ & $3112(1)$ & $5640(1)$ & $23(1)$ \\
$\mathrm{C} 2$ & $4755(1)$ & $3730(1)$ & $5382(1)$ & $20(1)$ \\
C3 & $6113(1)$ & $4063(1)$ & $5325(1)$ & $20(1)$ \\
C4 & $3735(1)$ & $4640(1)$ & $5061(1)$ & $21(1)$ \\
C5 & $3626(1)$ & $1840(1)$ & $6030(1)$ & $24(1)$ \\
C6 & $4181(1)$ & $473(1)$ & $6484(1)$ & $32(1)$ \\
C7 & $2294(1)$ & $1726(1)$ & $4806(1)$ & $31(1)$ \\
C8 & $3372(1)$ & $2528(1)$ & $7107(1)$ & $34(1)$ \\
C9 & $8380(1)$ & $3180(1)$ & $5679(1)$ & $26(1)$ \\
C10 & $8992(1)$ & $1826(1)$ & $6115(1)$ & $37(1)$ \\
C11 & $9272(1)$ & $4163(1)$ & $6686(1)$ & $38(1)$ \\
& $8359(1)$ & $3437(1)$ & $4341(1)$ & $39(1)$ \\
\hline
\end{tabular}


Table 3. Bond lengths $[\AA]$ and angles $\left[{ }^{\circ}\right]$ for 5.

\begin{tabular}{|c|c|c|c|}
\hline $\mathrm{N} 1-\mathrm{C} 1$ & $1.3487(12)$ & C6-H6B & $0.985(15)$ \\
\hline N1-C4 & $1.4696(12)$ & C6-H6C & $1.005(14)$ \\
\hline N1-H1N & $0.860(13)$ & C7-H7A & $0.977(14)$ \\
\hline $\mathrm{N} 2-\mathrm{C} 2$ & $1.2941(12)$ & C7-H7B & $0.979(16)$ \\
\hline $\mathrm{N} 2-\mathrm{C} 8$ & $1.4739(12)$ & C7-H7C & $0.988(15)$ \\
\hline $\mathrm{C} 1-\mathrm{C} 3$ & $1.3670(13)$ & C8-C10 & $1.5320(15)$ \\
\hline $\mathrm{C} 1-\mathrm{C} 2$ & $1.5128(12)$ & $\mathrm{C} 8-\mathrm{C} 11$ & $1.5332(16)$ \\
\hline C2-C3\#1 & $1.4372(13)$ & C8-C9 & $1.5344(14)$ \\
\hline C3-C2\#1 & $1.4372(13)$ & C9-H9A & $0.985(14)$ \\
\hline $\mathrm{C} 3-\mathrm{H} 3$ & $0.969(12)$ & C9-H9B & $0.994(16)$ \\
\hline $\mathrm{C} 4-\mathrm{C} 7$ & $1.5305(15)$ & C9-H9C & $0.983(15)$ \\
\hline $\mathrm{C} 4-\mathrm{C} 6$ & $1.5318(14)$ & C10-H10A & $0.995(14)$ \\
\hline $\mathrm{C} 4-\mathrm{C} 5$ & $1.5335(14)$ & C10-H10B & $0.976(15)$ \\
\hline $\mathrm{C} 5-\mathrm{H} 5 \mathrm{~A}$ & $0.961(13)$ & C10-H10C & $0.988(17)$ \\
\hline C5-H5B & $0.981(15)$ & C11-H11A & $0.955(13)$ \\
\hline C5-H5C & $0.980(14)$ & C11-H11B & $0.973(15)$ \\
\hline C6-H6A & $1.002(13)$ & C11-H11C & $0.994(14)$ \\
\hline C1-N1-C4 & $129.67(8)$ & N1-C4-C5 & $105.52(8)$ \\
\hline C1-N1-H1N & $111.6(8)$ & $\mathrm{C} 7-\mathrm{C} 4-\mathrm{C} 5$ & 109.09(9) \\
\hline C4-N1-H1N & 118.2(8) & C6-C4-C5 & $108.76(9)$ \\
\hline $\mathrm{C} 2-\mathrm{N} 2-\mathrm{C} 8$ & $124.93(8)$ & C4-C5-H5A & $112.7(8)$ \\
\hline N1-C1-C3 & $126.82(9)$ & C4-C5-H5B & $111.1(8)$ \\
\hline N1-C1-C2 & $113.00(8)$ & H5A-C5-H5B & $107.5(11)$ \\
\hline $\mathrm{C} 3-\mathrm{C} 1-\mathrm{C} 2$ & $120.18(8)$ & C4-C5-H5C & $108.7(8)$ \\
\hline N2-C2-C3\#1 & $129.20(8)$ & H5A-C5-H5C & $108.0(11)$ \\
\hline N2-C2-C1 & $113.89(8)$ & H5B-C5-H5C & $108.6(11)$ \\
\hline C3\#1-C2-C1 & $116.91(8)$ & C4-C6-H6A & $108.8(7)$ \\
\hline C1-C3-C2\#1 & $122.91(8)$ & C4-C6-H6B & $113.4(8)$ \\
\hline $\mathrm{C} 1-\mathrm{C} 3-\mathrm{H} 3$ & $120.3(7)$ & H6A-C6-H6B & $107.9(11)$ \\
\hline C2\#1-C3-H3 & $116.8(7)$ & C4-C6-H6C & $108.8(8)$ \\
\hline N1-C4-C7 & $110.97(8)$ & H6A-C6-H6C & $108.7(11)$ \\
\hline N1-C4-C6 & $111.22(8)$ & H6B-C6-H6C & $109.2(12)$ \\
\hline C7-C4-C6 & $111.08(9)$ & $\mathrm{C} 4-\mathrm{C} 7-\mathrm{H} 7 \mathrm{~A}$ & $109.6(8)$ \\
\hline
\end{tabular}




$\begin{array}{ll}\text { C4-C7-H7B } & 108.6(9) \\ \text { H7A-C7-H7B } & 108.7(12) \\ \text { C4-C7-H7C } & 111.2(8) \\ \text { H7A-C7-H7C } & 108.0(11) \\ \text { H7B-C7-H7C } & 110.8(12) \\ \text { N2-C8-C10 } & 111.40(9) \\ \text { N2-C8-C11 } & 111.88(9) \\ \text { C10-C8-C11 } & 112.26(10) \\ \text { N2-C8-C9 } & 104.92(8) \\ \text { C10-C8-C9 } & 107.97(9) \\ \text { C11-C8-C9 } & 108.01(9) \\ \text { C8-C9-H9A } & 109.5(8) \\ \text { C8-C9-H9B } & 109.0(9) \\ \text { H9A-C9-H9B } & 109.3(12) \\ \text { C8-C9-H9C } & 108.8(9) \\ \text { H9A-C9-H9C } & 110.4(12) \\ \text { H9B-C9-H9C } & 109.8(12) \\ \text { C8-C10-H10A } & 109.2(8) \\ \text { C8-C10-H10B } & 111.7(9) \\ \text { H10A-C10-H10B } & 111.2(12) \\ \text { C8-C10-H10C } & 108.9(9) \\ \text { H10A-C10-H10C } & 108.5(12) \\ \text { H10B-C10-H10C } & 107.2(13) \\ \text { C8-C11-H11A } & 107.7(7) \\ \text { C8-C11-H11B } & 112.4(9) \\ \text { H11A-C11-H11B } & 110.0(11) \\ \text { C8-C11-H11C } & 108.2(8) \\ \text { H11A-C11-H11C } & 109.2(11) \\ \text { H11B-C11-H11C } & 109.1(12) \\ & \end{array}$


Symmetry transformations used to generate equivalent atoms:

$\# 1-\mathrm{x}+1,-\mathrm{y}+1,-\mathrm{z}+1$ 
Table 4. Anisotropic displacement parameters $\left(\AA^{2} \times 10^{3}\right)$ for 5. The anisotropic displacement factor exponent takes the form: $-2 \pi^{2}\left[\mathrm{~h}^{2} \mathrm{a}^{* 2} \mathrm{U}^{11}+\ldots+2 \mathrm{hk} \mathrm{a}^{*} \mathrm{~b}^{*} \mathrm{U}^{12}\right]$

\begin{tabular}{lcccccc}
\hline & $\mathrm{U}^{11}$ & $\mathrm{U}^{22}$ & $\mathrm{U}^{33}$ & $\mathrm{U}^{23}$ & $\mathrm{U} 13$ & $\mathrm{U}^{12}$ \\
\hline $\mathrm{N} 1$ & $22(1)$ & $20(1)$ & $34(1)$ & $6(1)$ & $13(1)$ & $1(1)$ \\
$\mathrm{N} 2$ & $21(1)$ & $21(1)$ & $27(1)$ & $1(1)$ & $9(1)$ & $0(1)$ \\
$\mathrm{C} 1$ & $21(1)$ & $20(1)$ & $19(1)$ & $0(1)$ & $7(1)$ & $-3(1)$ \\
$\mathrm{C} 2$ & $20(1)$ & $21(1)$ & $18(1)$ & $-1(1)$ & $6(1)$ & $-2(1)$ \\
$\mathrm{C} 3$ & $19(1)$ & $21(1)$ & $23(1)$ & $1(1)$ & $9(1)$ & $-2(1)$ \\
$\mathrm{C} 4$ & $25(1)$ & $22(1)$ & $26(1)$ & $4(1)$ & $11(1)$ & $-3(1)$ \\
$\mathrm{C} 5$ & $34(1)$ & $25(1)$ & $36(1)$ & $10(1)$ & $13(1)$ & $-1(1)$ \\
$\mathrm{C} 6$ & $27(1)$ & $28(1)$ & $33(1)$ & $3(1)$ & $7(1)$ & $-5(1)$ \\
$\mathrm{C} 7$ & $45(1)$ & $33(1)$ & $31(1)$ & $2(1)$ & $21(1)$ & $-3(1)$ \\
$\mathrm{C} 8$ & $21(1)$ & $24(1)$ & $35(1)$ & $3(1)$ & $11(1)$ & $2(1)$ \\
$\mathrm{C} 9$ & $30(1)$ & $28(1)$ & $58(1)$ & $10(1)$ & $21(1)$ & $8(1)$ \\
$\mathrm{C} 10$ & $24(1)$ & $33(1)$ & $47(1)$ & $-1(1)$ & $5(1)$ & $-3(1)$ \\
$\mathrm{C} 11$ & $37(1)$ & $43(1)$ & $44(1)$ & $8(1)$ & $25(1)$ & $9(1)$ \\
& & & & & & \\
\hline
\end{tabular}


Table 5. Hydrogen coordinates $\left(\mathrm{x} 10^{4}\right)$ and isotropic displacement parameters $\left(\AA^{2} \times 10^{3}\right)$ for 5.

\begin{tabular}{|c|c|c|c|c|}
\hline & $\mathrm{x}$ & $\mathrm{y}$ & z & $\mathrm{U}(\mathrm{eq})$ \\
\hline $\mathrm{H} 1 \mathrm{~N}$ & $5480(13)$ & $2109(12)$ & $5969(12)$ & $30(3)$ \\
\hline H3 & $2869(12)$ & $4431(11)$ & $5105(11)$ & $25(3)$ \\
\hline H5A & $4344(13)$ & $-12(12)$ & $5837(13)$ & $35(3)$ \\
\hline H5B & $5050(15)$ & $507(13)$ & $7254(14)$ & $47(4)$ \\
\hline $\mathrm{H} 5 \mathrm{C}$ & $3505(14)$ & $-3(13)$ & $6696(13)$ & $38(3)$ \\
\hline H6A & $1599(13)$ & $1236(13)$ & $5016(12)$ & $38(3)$ \\
\hline H6B & $1890(14)$ & $2572(14)$ & $4444(14)$ & $45(4)$ \\
\hline $\mathrm{H} 6 \mathrm{C}$ & $2490(14)$ & $1227(14)$ & $4139(14)$ & $45(4)$ \\
\hline H7A & $2728(15)$ & 2021(13) & $7334(14)$ & $45(4)$ \\
\hline H7B & $4247(16)$ & $2590(14)$ & $7867(15)$ & $50(4)$ \\
\hline $\mathrm{H} 7 \mathrm{C}$ & $2970(14)$ & $3397(14)$ & $6830(14)$ & $45(4)$ \\
\hline H9A & $9947(15)$ & $1808(13)$ & 6193(13) & $45(4)$ \\
\hline H9B & $8438(16)$ & $1178(16)$ & $5460(15)$ & $55(4)$ \\
\hline $\mathrm{H} 9 \mathrm{C}$ & $8963(15)$ & $1629(14)$ & $6951(15)$ & $51(4)$ \\
\hline $\mathrm{H} 10 \mathrm{~A}$ & $10244(15)$ & $4076(14)$ & $6794(14)$ & $48(4)$ \\
\hline H10B & $8939(15)$ & $5048(15)$ & $6452(14)$ & $51(4)$ \\
\hline $\mathrm{H} 10 \mathrm{C}$ & $9228(16)$ & $3969(15)$ & $7520(16)$ & $58(4)$ \\
\hline H11A & $7752(13)$ & 2819(13) & $3757(12)$ & $31(3)$ \\
\hline H11B & $8059(15)$ & $4313(15)$ & $4037(15)$ & $52(4)$ \\
\hline $\mathrm{H} 11 \mathrm{C}$ & $9304(15)$ & $3302(13)$ & $4392(13)$ & $49(4)$ \\
\hline
\end{tabular}


Table 6. Torsion angles $\left[^{\circ}\right]$ for 5.

\begin{tabular}{lc}
\hline C4-N1-C1-C3 & $-4.98(16)$ \\
C4-N1-C1-C2 & $175.12(9)$ \\
C8-N2-C2-C3\#1 & $0.57(16)$ \\
C8-N2-C2-C1 & $-179.29(8)$ \\
N1-C1-C2-N2 & $0.55(12)$ \\
C3-C1-C2-N2 & $-179.36(8)$ \\
N1-C1-C2-C3\#1 & $-179.32(8)$ \\
C3-C1-C2-C3\#1 & $0.77(14)$ \\
N1-C1-C3-C2\#1 & $179.29(9)$ \\
C2-C1-C3-C2\#1 & $-0.81(15)$ \\
C1-N1-C4-C7 & $-59.60(13)$ \\
C1-N1-C4-C6 & $64.59(13)$ \\
C1-N1-C4-C5 & $-177.63(10)$ \\
C2-N2-C8-C10 & $62.92(13)$ \\
C2-N2-C8-C11 & $-63.65(13)$ \\
C2-N2-C8-C9 & $179.49(10)$ \\
\end{tabular}

Symmetry transformations used to generate equivalent atoms:

$\# 1-\mathrm{x}+1,-\mathrm{y}+1,-\mathrm{z}+1$ 
X-ray Crystallographic Data for 8:<smiles>CNc1cc(NS(C)(=O)=O)c(NNC(=O)[O-])cc1N</smiles>

Figure 3. View of 8 showing the atom labeling scheme. Displacement ellipsoids are scaled to the $50 \%$ probability level. The molecule lies on a crystallographic two-fold rotation axis at $1 / 2, y$, $1 / 4$. Atoms with labels appended by a ' are related by $1-x, y, 1 / 2-z$.

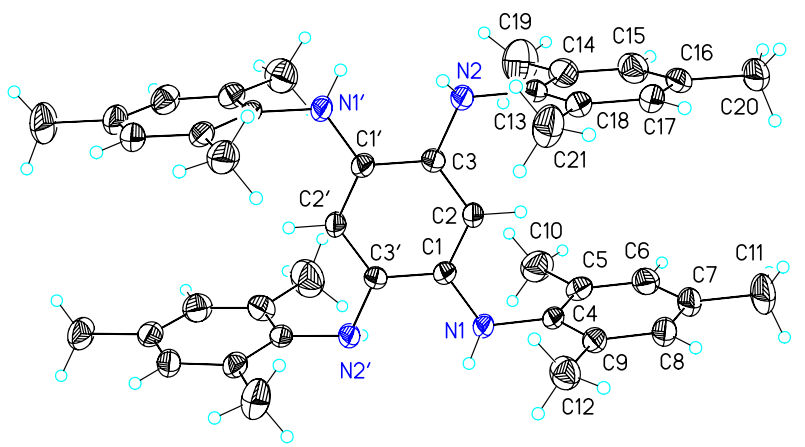

X-ray Experimental for $8\left(\mathrm{C}_{42} \mathrm{H}_{30} \mathrm{~N}_{4} \mathrm{O}_{4}-\mathrm{C}_{7} \mathrm{H}_{8}\right)$ : Crystals grew as nicely formed yellow-brown prisms lathes by slow evaporation from toluene. The data crystal was a prism that had approximate dimensions; $0.34 \times 0.33 \times 0.23 \mathrm{~mm}$. The data were collected on a Nonius Kappa CCD diffractometer using a graphite monochromator with $\mathrm{MoK} \alpha$ radiation $(\lambda=0.71073 \AA)$. A total of 269 frames of data were collected using $\omega$-scans with a scan range of $1.5^{\circ}$ and a counting time of 116 seconds per frame. The data were collected at $153 \mathrm{~K}$ using an Oxford Cryostream low temperature device. Details of crystal data, data collection and structure refinement are listed in Table 1. Data reduction were performed using DENZO-SMN. ${ }^{1}$ The structure was solved by direct methods using SIR97 $7^{2}$ and refined by full-matrix least-squares on $\mathrm{F}^{2}$ with anisotropic displacement parameters for the non-H atoms using SHELXL-97. ${ }^{3}$ The hydrogen atoms on carbon were calculated in ideal positions with isotropic displacement parameters set to $1.2 \mathrm{xUeq}$ of the attached atom (1.5xUeq for methyl hydrogen atoms). 
The molecule of toluene was disordered about a crystallographic two-fold rotation axis at $1 / 2, y, 1 / 4$. The toluene molecule was refined with half-weighted carbon atoms initially. The molecule was refined anisotropically as a rigid group with displacement parameters restrained to be approximately isotropic. The ammine also resided around the crystallographic two-fold rotation axis at $1 / 2, y, 1 / 4$. The hydrogen atoms bound to nitrogen appeared to be disordered. For each nitrogen atom, two peaks in a reasonable position to be a $\mathrm{H}$ atom appeared in the $\Delta \mathrm{F}$ map. In the final refinement model, two half-weighted hydrogen atoms were refined with isotropic displacement parameters.

The function, $\operatorname{\Sigma w}\left(\left|\mathrm{F}_{\mathrm{o}}\right|^{2}-\left|\mathrm{F}_{\mathrm{c}}\right|^{2}\right)^{2}$, was minimized, where $\mathrm{w}=1 /\left[\left(\sigma\left(\mathrm{F}_{\mathrm{o}}\right)\right)^{2}+(0.0719 * \mathrm{P})^{2}+\right.$ $(1.3529 * \mathrm{P})]$ and $\mathrm{P}=\left(\left|\mathrm{F}_{\mathrm{o}}\right|^{2}+2\left|\mathrm{~F}_{\mathrm{c}}\right|^{2}\right) / 3 . \mathrm{R}_{\mathrm{W}}\left(\mathrm{F}^{2}\right)$ refined to 0.176 , with $\mathrm{R}(\mathrm{F})$ equal to 0.0624 and a goodness of fit, $S,=1.08$. Definitions used for calculating $R(F), R_{W}\left(F^{2}\right)$ and the goodness of fit, $\mathrm{S}$, are given below. ${ }^{4}$ The data were checked for secondary extinction effects but no correction was necessary. Neutral atom scattering factors and values used to calculate the linear absorption coefficient are from the International Tables for X-ray Crystallography (1992). ${ }^{5}$ All figures were generated using SHELXTL/PC. ${ }^{6}$ Tables of positional and thermal parameters, bond lengths and angles, torsion angles, figures and lists of observed and calculated structure factors are located in tables 1 through 7.

\section{$\underline{\text { References }}$}

11) DENZO-SMN. (1997). Z. Otwinowski and W. Minor, Methods in Enzymology, 276: Macromolecular Crystallography, part A, 307 - 326, C. W. Carter, Jr. and R. M. Sweets, Editors, Academic Press.

12) SIR97. (1999). A program for crystal structure solution. Altomare A., Burla M.C., Camalli M., Cascarano G.L., Giacovazzo C. , Guagliardi A., Moliterni A.G.G., Polidori G.,Spagna R. J. Appl. Cryst. 32, 115-119.

13) Sheldrick, G. M. (1994). SHELXL97. Program for the Refinement of Crystal Structures. University of Gottingen, Germany.

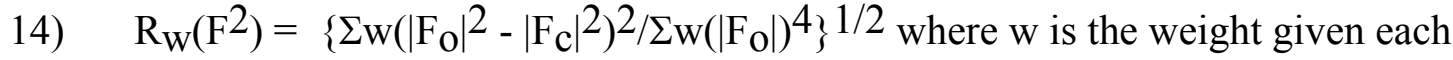
reflection. 
$\left.\mathrm{R}(\mathrm{F})=\Sigma\left(\left|\mathrm{F}_{\mathrm{O}}\right|-\left|\mathrm{F}_{\mathrm{C}}\right|\right) / \Sigma\left|\mathrm{F}_{\mathrm{O}}\right|\right\}$ for reflections with $\mathrm{F}_{\mathrm{O}}>4\left(\sigma\left(\mathrm{F}_{\mathrm{O}}\right)\right)$.

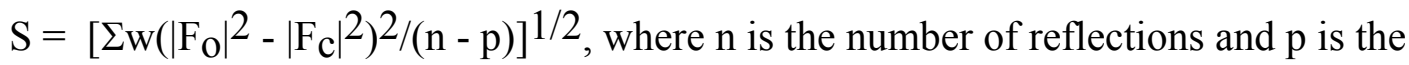
number of refined parameters.

15) International Tables for X-ray Crystallography (1992). Vol. C, Tables 4.2.6.8 and 6.1.1.4, A. J. C. Wilson, editor, Boston: Kluwer Academic Press.

6) Sheldrick, G. M. (1994). SHELXTL/PC (Version 5.03). Siemens Analytical Xray Instruments, Inc., Madison, Wisconsin, USA. 
Table 1. Crystal data and structure refinement for $\mathbf{8}$.

Empirical formula

Formula weight

Temperature

Wavelength

Crystal system

Space group

Unit cell dimensions

Volume

Z

Density (calculated)

Absorption coefficient

$\mathrm{F}(000)$

Crystal size

Theta range for data collection

Index ranges

Reflections collected

Independent reflections

Completeness to theta $=27.48^{\circ}$

Absorption correction

Refinement method

Data / restraints / parameters

Goodness-of-fit on $\mathrm{F}^{2}$

Final R indices $[\mathrm{I}>2 \operatorname{sigma}(\mathrm{I})]$

$\mathrm{R}$ indices (all data)

Largest diff. peak and hole
C49 H58 N4

702.99

153(2) K

$0.71073 \AA$

Monoclinic

$\mathrm{P} 2 / \mathrm{c}$

$\mathrm{a}=8.7265(1) \AA \quad \alpha=90^{\circ}$.

$\mathrm{b}=11.1041(2) \AA \quad \beta=96.666(1)^{\circ}$.

$\mathrm{c}=21.5922(3) \AA \quad \gamma=90^{\circ}$.

2078.14(5) $\AA^{3}$

2

$1.123 \mathrm{Mg} / \mathrm{m}^{3}$

$0.065 \mathrm{~mm}^{-1}$

760

$0.34 \times 0.33 \times 0.23 \mathrm{~mm}$

2.64 to $27.48^{\circ}$.

$-11<=\mathrm{h}<=11,-14<=\mathrm{k}<=14,-28<=\mathrm{l}<=27$

9143

$4746[\mathrm{R}(\mathrm{int})=0.0199]$

$99.3 \%$

None

Full-matrix least-squares on $\mathrm{F}^{2}$

4746 / 42 / 278

1.015

$\mathrm{R} 1=0.0624, \mathrm{wR} 2=0.1565$

$\mathrm{R} 1=0.0846, \mathrm{wR} 2=0.1761$

0.530 and -0.389 e. $\AA^{-3}$ 
Table 2. Atomic coordinates ( x 104) and equivalent isotropic displacement parameters $\left(\AA^{2} \mathrm{x}\right.$ $10^{3}$ ) for 8 . U(eq) is defined as one third of the trace of the orthogonalized Uij tensor.

\begin{tabular}{|c|c|c|c|c|}
\hline & $\mathrm{x}$ & $\mathrm{y}$ & zU(eq) & \\
\hline N1 & $7390(2)$ & $7174(2)$ & $3497(1)$ & $34(1)$ \\
\hline N2 & $1865(2)$ & $7157(2)$ & $2714(1)$ & $34(1)$ \\
\hline $\mathrm{C} 1$ & $6181(2)$ & $7165(2)$ & $3002(1)$ & $28(1)$ \\
\hline $\mathrm{C} 2$ & $4639(2)$ & $7159(2)$ & $3107(1)$ & $29(1)$ \\
\hline $\mathrm{C} 3$ & $3450(2)$ & $7158(2)$ & $2614(1)$ & $28(1)$ \\
\hline $\mathrm{C} 4$ & $7004(2)$ & $7303(2)$ & $4122(1)$ & $28(1)$ \\
\hline $\mathrm{C} 5$ & $6811(2)$ & $8451(2)$ & $4363(1)$ & $33(1)$ \\
\hline C6 & $6424(2)$ & $8552(2)$ & $4972(1)$ & $37(1)$ \\
\hline $\mathrm{C} 7$ & $6235(2)$ & $7548(2)$ & $5337(1)$ & $36(1)$ \\
\hline $\mathrm{C} 8$ & $6428(2)$ & $6416(2)$ & $5082(1)$ & $33(1)$ \\
\hline C9 & $6804(2)$ & $6275(2)$ & $4475(1)$ & $29(1)$ \\
\hline $\mathrm{C} 10$ & $6992(3)$ & $9550(2)$ & $3973(1)$ & $50(1)$ \\
\hline C11 & $5832(3)$ & $7676(2)$ & $5995(1)$ & $55(1)$ \\
\hline $\mathrm{C} 12$ & $6959(2)$ & $5038(2)$ & $4206(1)$ & $40(1)$ \\
\hline C13 & $1516(2)$ & $7291(2)$ & $3344(1)$ & $29(1)$ \\
\hline $\mathrm{C} 14$ & $1471(2)$ & $8436(2)$ & $3605(1)$ & $38(1)$ \\
\hline C15 & $1159(2)$ & $8544(2)$ & $4224(1)$ & $41(1)$ \\
\hline C16 & $898(2)$ & $7549(2)$ & $4584(1)$ & $34(1)$ \\
\hline $\mathrm{C} 17$ & $947(2)$ & $6424(2)$ & $4310(1)$ & $33(1)$ \\
\hline C18 & $1251(2)$ & $6267(2)$ & $3693(1)$ & $31(1)$ \\
\hline C19 & $1792(3)$ & $9537(2)$ & $3234(1)$ & $65(1)$ \\
\hline $\mathrm{C} 20$ & $561(3)$ & $7684(2)$ & $5250(1)$ & $46(1)$ \\
\hline $\mathrm{C} 21$ & $1296(3)$ & $5026(2)$ & $3422(1)$ & $50(1)$ \\
\hline $\mathrm{C} 1 \mathrm{~A}$ & $6093(5)$ & $2548(3)$ & $2644(2)$ & $83(2)$ \\
\hline $\mathrm{C} 2 \mathrm{~A}$ & $5273(6)$ & $1543(3)$ & $2513(3)$ & $49(2)$ \\
\hline $\mathrm{C} 3 \mathrm{~A}$ & $3555(5)$ & $1792(4)$ & $2292(2)$ & $75(2)$ \\
\hline $\mathrm{C} 4 \mathrm{~A}$ & $3003(6)$ & $2965(5)$ & $2229(3)$ & $105(3)$ \\
\hline C5A & $3995(8)$ & $3929(4)$ & $2373(4)$ & $126(4)$ \\
\hline C6A & $5541(7)$ & $3721(3)$ & $2580(4)$ & $84(3)$ \\
\hline C7A & $7766(5)$ & $2395(6)$ & 2881(3) & $131(4)$ \\
\hline
\end{tabular}


Table 3. Bond lengths $[\AA]$ and angles $\left[{ }^{\circ}\right]$ for 8.

\begin{tabular}{|c|c|c|c|}
\hline $\mathrm{N} 1-\mathrm{C} 1$ & $1.413(2)$ & C13-C18 & $1.399(3)$ \\
\hline N1-C4 & $1.435(2)$ & C14-C15 & $1.400(3)$ \\
\hline N1-H1NB & $0.92(5)$ & C14-C19 & $1.506(3)$ \\
\hline N1-H1NA & $0.82(4)$ & $\mathrm{C} 15-\mathrm{C} 16$ & $1.384(3)$ \\
\hline N2-C3 & $1.424(2)$ & C15-H15 & 0.95 \\
\hline N2-C13 & $1.435(2)$ & $\mathrm{C} 16-\mathrm{C} 17$ & $1.385(3)$ \\
\hline N2-H2NB & $0.93(4)$ & $\mathrm{C} 16-\mathrm{C} 20$ & $1.509(3)$ \\
\hline N2-H2NA & $0.88(6)$ & C17-C18 & $1.397(2)$ \\
\hline $\mathrm{C} 1-\mathrm{C} 2$ & $1.391(2)$ & C17-H17 & 0.95 \\
\hline C1-C3\#1 & $1.404(2)$ & C18-C21 & $1.499(3)$ \\
\hline $\mathrm{C} 2-\mathrm{C} 3$ & $1.398(2)$ & C19-H19A & 0.98 \\
\hline C2-H2 & 0.95 & C19-H19B & 0.98 \\
\hline C3-C1\#1 & $1.404(2)$ & C19-H19C & 0.98 \\
\hline C4-C5 & $1.395(3)$ & C20-H20A & 0.98 \\
\hline C4-C9 & $1.396(3)$ & C20-H20B & 0.98 \\
\hline $\mathrm{C} 5-\mathrm{C} 6$ & $1.400(3)$ & C20-H20C & 0.98 \\
\hline C5-C10 & $1.503(3)$ & C21-H21A & 0.98 \\
\hline C6-C7 & $1.386(3)$ & C21-H21B & 0.98 \\
\hline C6-H6 & 0.95 & C21-H21C & 0.98 \\
\hline $\mathrm{C} 7-\mathrm{C} 8$ & $1.390(3)$ & $\mathrm{C} 1 \mathrm{~A}-\mathrm{C} 2 \mathrm{~A}$ & 1.338 \\
\hline C7-C11 & $1.511(3)$ & C1A-C6A & 1.390 \\
\hline C8-C9 & $1.396(2)$ & C1A-C7A & 1.500 \\
\hline C8-H8 & 0.95 & $\mathrm{C} 2 \mathrm{~A}-\mathrm{C} 3 \mathrm{~A}$ & 1.545 \\
\hline C9-C12 & $1.503(3)$ & $\mathrm{C} 2 \mathrm{~A}-\mathrm{H} 2 \mathrm{AA}$ & 0.960 \\
\hline C10-H10A & 0.98 & $\mathrm{C} 3 \mathrm{~A}-\mathrm{C} 4 \mathrm{~A}$ & 1.390 \\
\hline C10-H10B & 0.98 & C3A-H3AA & 0.96 \\
\hline C10-H10C & 0.98 & C4A-C5A & 1.390 \\
\hline C11-H11A & 0.98 & C4A-H4AA & 0.96 \\
\hline C11-H11B & 0.98 & C5A-C6A & 1.390 \\
\hline $\mathrm{C} 11-\mathrm{H} 11 \mathrm{C}$ & 0.98 & C5A-H5AA & 0.96 \\
\hline $\mathrm{C} 12-\mathrm{H} 12 \mathrm{~A}$ & 0.98 & C6A-H6AA & 0.96 \\
\hline C12-H12B & 0.98 & C7A-H7AA & 0.96 \\
\hline $\mathrm{C} 12-\mathrm{H} 12 \mathrm{C}$ & 0.98 & C7A-H7AB & 0.96 \\
\hline C13-C14 & $1.393(3)$ & C7A-H7AC & 0.96 \\
\hline
\end{tabular}




\begin{tabular}{|c|c|c|c|}
\hline C1-N1-C4 & $118.42(14)$ & C4-C9-C12 & $120.91(15)$ \\
\hline C1-N1-H1NB & $110(3)$ & C8-C9-C12 & $120.44(17)$ \\
\hline C4-N1-H1NB & 111(3) & C5-C10-H10A & 109.5 \\
\hline C1-N1-H1NA & $120(3)$ & C5-C10-H10B & 109.5 \\
\hline C4-N1-H1NA & $118(3)$ & H10A-C10-H10B & 109.5 \\
\hline C3-N2-C13 & $117.36(14)$ & C5-C10-H10C & 109.5 \\
\hline $\mathrm{C} 3-\mathrm{N} 2-\mathrm{H} 2 \mathrm{NB}$ & $107(2)$ & H10A-C10-H10C & 109.5 \\
\hline C13-N2-H2NB & $95(2)$ & H10B-C10-H10C & 109.5 \\
\hline C3-N2-H2NA & $114(3)$ & C7-C11-H11A & 109.5 \\
\hline C13-N2-H2NA & 115(3) & C7-C11-H11B & 109.5 \\
\hline C2-C1-C3\#1 & $119.26(15)$ & H11A-C11-H11B & 109.5 \\
\hline $\mathrm{C} 2-\mathrm{C} 1-\mathrm{N} 1$ & $121.83(15)$ & C7-C11-H11C & 109.5 \\
\hline C3\#1-C1-N1 & $118.91(14)$ & $\mathrm{H} 11 \mathrm{~A}-\mathrm{C} 11-\mathrm{H} 11 \mathrm{C}$ & 109.5 \\
\hline $\mathrm{C} 1-\mathrm{C} 2-\mathrm{C} 3$ & $121.43(15)$ & H11B-C11-H11C & 109.5 \\
\hline $\mathrm{C} 1-\mathrm{C} 2-\mathrm{H} 2$ & 119.3 & C9-C12-H12A & 109.5 \\
\hline $\mathrm{C} 3-\mathrm{C} 2-\mathrm{H} 2$ & 119.3 & C9-C12-H12B & 109.5 \\
\hline $\mathrm{C} 2-\mathrm{C} 3-\mathrm{C} 1 \# 1$ & $119.31(15)$ & H12A-C12-H12B & 109.5 \\
\hline $\mathrm{C} 2-\mathrm{C} 3-\mathrm{N} 2$ & $122.21(15)$ & C9-C12-H12C & 109.5 \\
\hline $\mathrm{C} 1 \# 1-\mathrm{C} 3-\mathrm{N} 2$ & $118.48(14)$ & $\mathrm{H} 12 \mathrm{~A}-\mathrm{C} 12-\mathrm{H} 12 \mathrm{C}$ & 109.5 \\
\hline $\mathrm{C} 5-\mathrm{C} 4-\mathrm{C} 9$ & $120.96(15)$ & H12B-C12-H12C & 109.5 \\
\hline $\mathrm{C} 5-\mathrm{C} 4-\mathrm{N} 1$ & $119.68(17)$ & C14-C13-C18 & $120.59(16)$ \\
\hline C9-C4-N1 & $119.35(17)$ & $\mathrm{C} 14-\mathrm{C} 13-\mathrm{N} 2$ & $119.82(17)$ \\
\hline $\mathrm{C} 4-\mathrm{C} 5-\mathrm{C} 6$ & $118.56(17)$ & $\mathrm{C} 18-\mathrm{C} 13-\mathrm{N} 2$ & $119.58(17)$ \\
\hline $\mathrm{C} 4-\mathrm{C} 5-\mathrm{C} 10$ & $120.46(17)$ & C13-C14-C15 & $118.85(18)$ \\
\hline C6-C5-C10 & $120.98(19)$ & C13-C14-C19 & $120.68(19)$ \\
\hline C7-C6-C5 & $121.73(18)$ & C15-C14-C19 & $120.5(2)$ \\
\hline C7-C6-H6 & 119.1 & C16-C15-C14 & $122.02(19)$ \\
\hline C5-C6-H6 & 119.1 & C16-C15-H15 & 119.0 \\
\hline C6-C7-C8 & $118.40(17)$ & C14-C15-H15 & 119.0 \\
\hline C6-C7-C11 & $120.94(19)$ & C15-C16-C17 & $117.66(17)$ \\
\hline C8-C7-C11 & $120.66(18)$ & $\mathrm{C} 15-\mathrm{C} 16-\mathrm{C} 20$ & $121.24(18)$ \\
\hline C7-C8-C9 & $121.70(18)$ & C17-C16-C20 & $121.10(18)$ \\
\hline C7-C8-H8 & 119.2 & C16-C17-C18 & $122.60(18)$ \\
\hline C9-C8-H8 & 119.2 & C16-C17-H17 & 118.7 \\
\hline C4-C9-C8 & $118.64(17)$ & C18-C17-H17 & 118.7 \\
\hline
\end{tabular}




\begin{tabular}{|c|c|c|c|}
\hline C17-C18-C13 & $118.28(17)$ & C1A-C6A-C5A & 120.0 \\
\hline C17-C18-C21 & $120.27(18)$ & C1A-C6A-H6AA & 120.0 \\
\hline C13-C18-C21 & $121.45(16)$ & C5A-C6A-H6AA & 120.0 \\
\hline C14-C19-H19A & 109.5 & C1A-C7A-H7AA & 109.5 \\
\hline C14-C19-H19B & 109.5 & C1A-C7A-H7AB & 109.5 \\
\hline H19A-C19-H19B & 109.5 & H7AA-C7A-H7AB & 109.5 \\
\hline C14-C19-H19C & 109.5 & C1A-C7A-H7AC & 109.5 \\
\hline H19A-C19-H19C & 109.5 & H7AA-C7A-H7AC & 109.5 \\
\hline H19B-C19-H19C & 109.5 & H7AB-C7A-H7AC & 109.5 \\
\hline C16-C20-H20A & 109.5 & & \\
\hline $\mathrm{C} 16-\mathrm{C} 20-\mathrm{H} 20 \mathrm{~B}$ & 109.5 & & \\
\hline H20A-C20-H20B & 109.5 & & \\
\hline C16-C20-H20C & 109.5 & & \\
\hline $\mathrm{H} 20 \mathrm{~A}-\mathrm{C} 20-\mathrm{H} 20 \mathrm{C}$ & 109.5 & & \\
\hline H20B-C20-H20C & 109.5 & & \\
\hline $\mathrm{C} 18-\mathrm{C} 21-\mathrm{H} 21 \mathrm{~A}$ & 109.5 & & \\
\hline C18-C21-H21B & 109.5 & & \\
\hline H21A-C21-H21B & 109.5 & & \\
\hline $\mathrm{C} 18-\mathrm{C} 21-\mathrm{H} 21 \mathrm{C}$ & 109.5 & & \\
\hline $\mathrm{H} 21 \mathrm{~A}-\mathrm{C} 21-\mathrm{H} 21 \mathrm{C}$ & 109.5 & & \\
\hline $\mathrm{H} 21 \mathrm{~B}-\mathrm{C} 21-\mathrm{H} 21 \mathrm{C}$ & 109.5 & & \\
\hline C2A-C1A-C6A & 126.2 & & \\
\hline $\mathrm{C} 2 \mathrm{~A}-\mathrm{C} 1 \mathrm{~A}-\mathrm{C} 7 \mathrm{~A}$ & 116.9 & & \\
\hline C6A-C1A-C7A & 116.9 & & \\
\hline C1A-C2A-C3A & 113.1 & & \\
\hline C1A-C2A-H2AA & 123.5 & & \\
\hline C3A-C2A-H2AA & 123.4 & & \\
\hline C4A-C3A-C2A & 120.7 & & \\
\hline C4A-C3A-H3AA & 119.6 & & \\
\hline C2A-C3A-H3AA & 119.6 & & \\
\hline C5A-C4A-C3A & 120.0 & & \\
\hline C5A-C4A-H4AA & 120.0 & & \\
\hline C3A-C4A-H4AA & 120.0 & & \\
\hline C4A-C5A-C6A & 120.0 & & \\
\hline C4A-C5A-H5AA & 120.0 & & \\
\hline C6A-C5A-H5AA & 120.0 & & \\
\hline
\end{tabular}


Symmetry transformations used to generate equivalent atoms:

\#1-x+1,y,-z+1/2 
Table 4. Anisotropic displacement parameters $\left(\AA^{2} \times 10^{3}\right)$ for 8 . The anisotropic displacement factor exponent takes the form: $-2 \pi^{2}\left[\mathrm{~h}^{2} \mathrm{a}^{* 2} \mathrm{U}^{11}+\ldots+2 \mathrm{~h} \mathrm{k} \mathrm{a}^{*} \mathrm{~b}^{*} \mathrm{U}^{12}\right]$

\begin{tabular}{|c|c|c|c|c|c|c|}
\hline & U11 & $\mathrm{U}^{22}$ & $\mathrm{U}^{33}$ & $\mathrm{U}^{23}$ & $\mathrm{U}^{13} \mathrm{U}^{12}$ & \\
\hline N1 & $19(1)$ & $66(1)$ & $18(1)$ & $2(1)$ & $1(1)$ & $3(1)$ \\
\hline $\mathrm{N} 2$ & $19(1)$ & $62(1)$ & $20(1)$ & $2(1)$ & $3(1)$ & $-2(1)$ \\
\hline $\mathrm{C} 1$ & $20(1)$ & $43(1)$ & $21(1)$ & $0(1)$ & $0(1)$ & $2(1)$ \\
\hline $\mathrm{C} 2$ & $22(1)$ & $48(1)$ & $18(1)$ & $0(1)$ & $4(1)$ & $1(1)$ \\
\hline $\mathrm{C} 3$ & $18(1)$ & $43(1)$ & $23(1)$ & $1(1)$ & $3(1)$ & $-2(1)$ \\
\hline $\mathrm{C} 4$ & $19(1)$ & $47(1)$ & $18(1)$ & $0(1)$ & $-1(1)$ & $2(1)$ \\
\hline C5 & $27(1)$ & $42(1)$ & $29(1)$ & $5(1)$ & $-2(1)$ & $3(1)$ \\
\hline C6 & $35(1)$ & $42(1)$ & $33(1)$ & $-7(1)$ & $2(1)$ & $6(1)$ \\
\hline $\mathrm{C} 7$ & $34(1)$ & $51(1)$ & $23(1)$ & $-5(1)$ & $4(1)$ & $2(1)$ \\
\hline $\mathrm{C} 8$ & $32(1)$ & $43(1)$ & $25(1)$ & $1(1)$ & $4(1)$ & $-1(1)$ \\
\hline C9 & $23(1)$ & $42(1)$ & $22(1)$ & $-4(1)$ & $0(1)$ & $0(1)$ \\
\hline $\mathrm{C} 10$ & $51(1)$ & $49(1)$ & $51(1)$ & $14(1)$ & $4(1)$ & $4(1)$ \\
\hline $\mathrm{C} 11$ & $72(2)$ & $65(2)$ & $29(1)$ & $-10(1)$ & $18(1)$ & $2(1)$ \\
\hline $\mathrm{C} 12$ & $41(1)$ & $45(1)$ & $36(1)$ & $-8(1)$ & $5(1)$ & $-1(1)$ \\
\hline $\mathrm{C} 13$ & $16(1)$ & $49(1)$ & $23(1)$ & $0(1)$ & $2(1)$ & $0(1)$ \\
\hline $\mathrm{C} 14$ & $31(1)$ & $45(1)$ & $38(1)$ & $3(1)$ & $5(1)$ & $-4(1)$ \\
\hline $\mathrm{C} 15$ & $37(1)$ & $46(1)$ & $39(1)$ & $-9(1)$ & $6(1)$ & $1(1)$ \\
\hline $\mathrm{C} 16$ & $21(1)$ & $53(1)$ & $27(1)$ & $-5(1)$ & $3(1)$ & $2(1)$ \\
\hline $\mathrm{C} 17$ & $29(1)$ & $46(1)$ & $24(1)$ & $4(1)$ & $3(1)$ & $1(1)$ \\
\hline $\mathrm{C} 18$ & $24(1)$ & $44(1)$ & $24(1)$ & $-1(1)$ & $1(1)$ & $2(1)$ \\
\hline C19 & $84(2)$ & $52(2)$ & $60(2)$ & $10(1)$ & $16(1)$ & $-14(1)$ \\
\hline $\mathrm{C} 20$ & $41(1)$ & $69(2)$ & $29(1)$ & $-10(1)$ & $7(1)$ & $5(1)$ \\
\hline $\mathrm{C} 21$ & $70(2)$ & $48(1)$ & $32(1)$ & $-3(1)$ & $6(1)$ & $5(1)$ \\
\hline $\mathrm{C} 1 \mathrm{~A}$ & $131(6)$ & $98(5)$ & $26(3)$ & $2(3)$ & $40(3)$ & $-12(4)$ \\
\hline $\mathrm{C} 2 \mathrm{~A}$ & $79(6)$ & $43(2)$ & $33(2)$ & $-2(3)$ & $33(3)$ & $-9(3)$ \\
\hline $\mathrm{C} 3 \mathrm{~A}$ & $133(6)$ & $65(4)$ & $36(3)$ & $-2(3)$ & $42(3)$ & $0(4)$ \\
\hline $\mathrm{C} 4 \mathrm{~A}$ & $134(7)$ & $148(8)$ & $41(3)$ & $12(5)$ & $41(4)$ & $38(6)$ \\
\hline $\mathrm{C} 5 \mathrm{~A}$ & 235(9) & $86(5)$ & $74(6)$ & $7(5)$ & $90(6)$ & $22(6)$ \\
\hline C6A & 181(9) & $43(3)$ & $40(4)$ & $-11(3)$ & $64(5)$ & $-35(4)$ \\
\hline C7A & $162(8)$ & $170(8)$ & $65(5)$ & $1(5)$ & $29(5)$ & $-39(7)$ \\
\hline
\end{tabular}


Table 5. Hydrogen coordinates ( $\left.\times 10^{4}\right)$ and isotropic displacement parameters $\left(\AA^{2} \times 10^{3}\right)$ for 8.

\begin{tabular}{|c|c|c|c|c|}
\hline & $\mathrm{x}$ & $\mathrm{y}$ & \multicolumn{2}{|c|}{ z U(eq) } \\
\hline $\mathrm{H} 2$ & 4388 & 7155 & 3524 & 35 \\
\hline H6 & 6289 & 9331 & 5141 & 44 \\
\hline H8 & 6300 & 5721 & 5327 & 40 \\
\hline $\mathrm{H} 10 \mathrm{~A}$ & 8026 & 9556 & 3837 & 76 \\
\hline H10B & 6853 & 10274 & 4219 & 76 \\
\hline $\mathrm{H} 10 \mathrm{C}$ & 6217 & 9537 & 3606 & 76 \\
\hline H11A & 5724 & 8532 & 6093 & 82 \\
\hline H11B & 6654 & 7321 & 6286 & 82 \\
\hline $\mathrm{H} 11 \mathrm{C}$ & 4858 & 7259 & 6034 & 82 \\
\hline $\mathrm{H} 12 \mathrm{~A}$ & 6799 & 4431 & 4522 & 61 \\
\hline H12B & 7993 & 4944 & 4078 & 61 \\
\hline $\mathrm{H} 12 \mathrm{C}$ & 6185 & 4932 & 3843 & 61 \\
\hline H15 & 1126 & 9324 & 4403 & 49 \\
\hline H17 & 768 & 5733 & 4550 & 40 \\
\hline H19A & 2895 & 9573 & 3188 & 97 \\
\hline H19B & 1495 & 10259 & 3452 & 97 \\
\hline H19C & 1195 & 9495 & 2821 & 97 \\
\hline $\mathrm{H} 20 \mathrm{~A}$ & 1480 & 7468 & 5533 & 69 \\
\hline H20B & -294 & 7152 & 5325 & 69 \\
\hline $\mathrm{H} 20 \mathrm{C}$ & 277 & 8521 & 5325 & 69 \\
\hline $\mathrm{H} 21 \mathrm{~A}$ & 1090 & 4431 & 3736 & 75 \\
\hline $\mathrm{H} 21 \mathrm{~B}$ & 2318 & 4878 & 3290 & 75 \\
\hline $\mathrm{H} 21 \mathrm{C}$ & 510 & 4960 & 3060 & 75 \\
\hline $\mathrm{H} 2 \mathrm{AA}$ & 5708 & 749 & 2553 & 59 \\
\hline H3AA & 2860 & 1129 & 2198 & 90 \\
\hline H4AA & 1935 & 3109 & 2086 & 126 \\
\hline H5AA & 3614 & 4740 & 2328 & 151 \\
\hline H6AA & 6227 & 4387 & 2679 & 101 \\
\hline H7AA & 8013 & 1552 & 2906 & 197 \\
\hline H7AB & 8388 & 2784 & 2602 & 197 \\
\hline
\end{tabular}




\begin{tabular}{lllll} 
H7AC & 7966 & 2749 & 3288 & 197 \\
H2NB & $1470(50)$ & $7900(40)$ & $2582(18)$ & $27(10)$ \\
H1NB & $8120(50)$ & $7730(40)$ & $3420(20)$ & $33(11)$ \\
H2NA & $1310(60)$ & $6610(50)$ & $2490(20)$ & $59(15)$ \\
H1NA & $8220(50)$ & $6860(40)$ & $3449(18)$ & $24(10)$ \\
\hline
\end{tabular}


Table 6. Torsion angles $\left[{ }^{\circ}\right]$ for 8.

\begin{tabular}{|c|c|}
\hline C4-N1-C1-C2 & $-6.5(3)$ \\
\hline C4-N1-C1-C3\#1 & $173.86(18)$ \\
\hline C3\#1-C1-C2-C3 & $-0.7(3)$ \\
\hline N1-C1-C2-C3 & 179.63(19) \\
\hline C1-C2-C3-C1\#1 & $-0.1(3)$ \\
\hline $\mathrm{C} 1-\mathrm{C} 2-\mathrm{C} 3-\mathrm{N} 2$ & $-179.69(19)$ \\
\hline C13-N2-C3-C2 & $6.7(3)$ \\
\hline C13-N2-C3-C1\#1 & $-172.89(18)$ \\
\hline C1-N1-C4-C5 & $-85.6(2)$ \\
\hline C1-N1-C4-C9 & $93.4(2)$ \\
\hline C9-C4-C5-C6 & $0.6(3)$ \\
\hline N1-C4-C5-C6 & 179.61(15) \\
\hline C9-C4-C5-C10 & $-178.54(17)$ \\
\hline N1-C4-C5-C10 & $0.5(3)$ \\
\hline C4-C5-C6-C7 & $0.1(3)$ \\
\hline C10-C5-C6-C7 & $179.23(18)$ \\
\hline C5-C6-C7-C8 & $-0.4(3)$ \\
\hline C5-C6-C7-C11 & 179.41(19) \\
\hline C6-C7-C8-C9 & $0.0(3)$ \\
\hline C11-C7-C8-C9 & $-179.79(18)$ \\
\hline C5-C4-C9-C8 & $-1.0(2)$ \\
\hline N1-C4-C9-C8 & $-179.97(15)$ \\
\hline C5-C4-C9-C12 & $177.85(16)$ \\
\hline N1-C4-C9-C12 & $-1.2(2)$ \\
\hline C7-C8-C9-C4 & $0.7(3)$ \\
\hline C7-C8-C9-C12 & $-178.17(17)$ \\
\hline C3-N2-C13-C14 & $83.0(2)$ \\
\hline C3-N2-C13-C18 & $-95.9(2)$ \\
\hline C18-C13-C14-C15 & $0.2(3)$ \\
\hline N2-C13-C14-C15 & $-178.71(16)$ \\
\hline C18-C13-C14-C19 & $178.58(19)$ \\
\hline N2-C13-C14-C19 & $-0.3(3)$ \\
\hline C13-C14-C15-C16 & $0.1(3)$ \\
\hline C19-C14-C15-C16 & $-178.3(2)$ \\
\hline
\end{tabular}




$\begin{array}{ll}\text { C14-C15-C16-C17 } & -0.3(3) \\ \text { C14-C15-C16-C20 } & -179.88(18) \\ \text { C15-C16-C17-C18 } & 0.2(3) \\ \text { C20-C16-C17-C18 } & 179.79(17) \\ \text { C16-C17-C18-C13 } & 0.1(3) \\ \text { C16-C17-C18-C21 } & 179.78(18) \\ \text { C14-C13-C18-C17 } & -0.2(3) \\ \text { N2-C13-C18-C17 } & 178.62(15) \\ \text { C14-C13-C18-C21 } & -179.94(18) \\ \text { N2-C13-C18-C21 } & -1.1(3) \\ \text { C6A-C1A-C2A-C3A } & -1.8 \\ \text { C7A-C1A-C2A-C3A } & 178.1 \\ \text { C1A-C2A-C3A-C4A } & 1.7 \\ \text { C3A-C4A-C5A-C6A } & 0.0 \\ \text { C2A-C1A-C6A-C5A } & 1.1 \\ \text { C7A-C1A-C6A-C5A } & -178.9 \\ \text { C4A-C5A-C6A-C1A } & 0.0\end{array}$

Symmetry transformations used to generate equivalent atoms:

$\# 1-\mathrm{x}+1, \mathrm{y},-\mathrm{z}+1 / 2$ 
X-ray Crystallographic Data for 9:

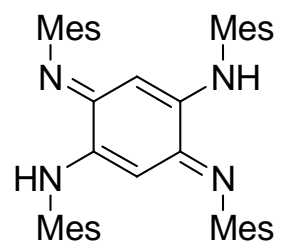

Figure 4. View of 9 showing the atom labeling scheme. Displacement ellipsoids are scaled to the $50 \%$ probability level. The methyl group hydrogen atoms have been removed for clarity. The molecule lies around a crystallographic inversion center at $1 / 2,1 / 2,1 / 2$. Atoms with labels appended by a ' are related by $1-\mathrm{x}, 1-\mathrm{y}, 1-\mathrm{z}$.

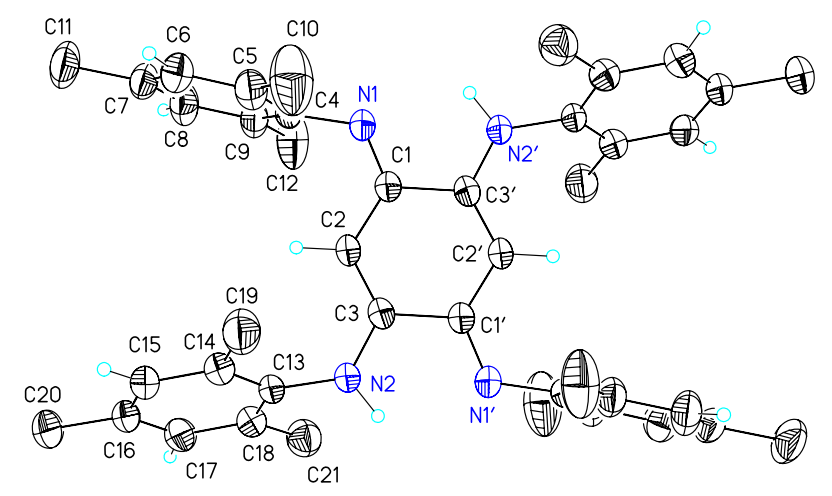

X-ray Experimental for $9\left(\mathrm{C}_{42} \mathrm{H}_{48} \mathrm{~N}_{4}\right)$ : Crystals grew as brown-orange prisms by slow evaporation from toluene. The data crystal was a prism that had approximate dimensions; $0.20 \mathrm{x}$ $0.14 \times 0.14 \mathrm{~mm}$. The data were collected on a Nonius Kappa CCD diffractometer using a graphite monochromator with $\mathrm{MoK} \alpha$ radiation $(\lambda=0.71073 \AA)$. A total of 372 frames of data were collected using $\omega$-scans with a scan range of $1.5^{\circ}$ and a counting time of 176 seconds per frame. The data were collected at $153 \mathrm{~K}$ using an Oxford Cryostream low temperature device. Details of crystal data, data collection and structure refinement are listed in Table 1. Data reduction were performed using DENZO-SMN. ${ }^{1}$ The structure was solved by direct methods using $\mathrm{SIR}^{2}$ and refined by full-matrix least-squares on $\mathrm{F}^{2}$ with anisotropic displacement parameters for the non-H atoms using SHELXL-97. ${ }^{3}$ The hydrogen atoms on the methyl carbon atoms were calculated in ideal positions with isotropic displacement parameters set to $1.5 \mathrm{xUeq}$ of the attached atom. The remaining hydrogen atoms were observed in a $\Delta \mathrm{F}$ map and refined 
with isotropic displacement parameters. The function, $\Sigma \mathrm{w}\left(\left|\mathrm{F}_{\mathrm{o}}\right|^{2}-\left|\mathrm{F}_{\mathrm{c}}\right|^{2}\right)^{2}$, was minimized, where $\mathrm{W}=1 /\left[\left(\sigma\left(\mathrm{F}_{\mathrm{o}}\right)\right)^{2}+(0.0465 * \mathrm{P})^{2}+(0.126 * \mathrm{P})\right]$ and $\mathrm{P}=\left(\left|\mathrm{F}_{\mathrm{o}}\right|^{2}+2\left|\mathrm{~F}_{\mathrm{c}}\right|^{2}\right) / 3 . \mathrm{R}_{\mathrm{W}}\left(\mathrm{F}^{2}\right)$ refined to 0.136 , with $\mathrm{R}(\mathrm{F})$ equal to 0.0522 and a goodness of fit, $\mathrm{S},=1.12$. Definitions used for calculating $\mathrm{R}(\mathrm{F})$, $\mathrm{R}_{\mathrm{W}}\left(\mathrm{F}^{2}\right)$ and the goodness of fit, $\mathrm{S}$, are given below. ${ }^{4}$ The data were corrected for secondary extinction effects. The correction takes the form: $\mathrm{F}_{\text {corr }}=\mathrm{kF}_{\mathrm{c}} /\left[1+\left(3.9(6) \times 10^{-5}\right)^{*} \mathrm{~F}_{\mathrm{c}}^{2}\right.$ $\left.\lambda^{3} /(\sin 2 \theta)\right]^{0.25}$ where $\mathrm{k}$ is the overall scale factor. Neutral atom scattering factors and values used to calculate the linear absorption coefficient are from the International Tables for X-ray Crystallography (1992). ${ }^{5}$ All figures were generated using SHELXTL/PC. 6 Tables of positional and thermal parameters, bond lengths and angles, torsion angles, figures and lists of observed and calculated structure factors are located in tables 1 through 7.

\section{References}

16) DENZO-SMN. (1997). Z. Otwinowski and W. Minor, Methods in Enzymology, 276: Macromolecular Crystallography, part A, 307 - 326, C. W. Carter, Jr. and R. M. Sweets, Editors, Academic Press.

17) SIR97. (1999). A program for crystal structure solution. Altomare A., Burla M.C., Camalli M., Cascarano G.L., Giacovazzo C. , Guagliardi A., Moliterni A.G.G., Polidori G.,Spagna R. J. Appl. Cryst. 32, 115-119.

18) Sheldrick, G. M. (1994). SHELXL97. Program for the Refinement of Crystal Structures. University of Gottingen, Germany.

19) $\mathrm{R}_{\mathrm{W}}\left(\mathrm{F}^{2}\right)=\left\{\sum \mathrm{w}\left(\left|\mathrm{F}_{\mathrm{O}}\right|^{2}-\left|\mathrm{F}_{\mathrm{c}}\right|^{2}\right)^{2 / \Sigma \mathrm{w}}\left(\left|\mathrm{F}_{\mathrm{O}}\right|\right)^{4}\right\}^{1 / 2}$ where $\mathrm{w}$ is the weight given each reflection.

$\left.\mathrm{R}(\mathrm{F})=\Sigma\left(\left|\mathrm{F}_{\mathrm{O}}\right|-\left|\mathrm{F}_{\mathrm{C}}\right|\right) / \Sigma\left|\mathrm{F}_{\mathrm{O}}\right|\right\}$ for reflections with $\mathrm{F}_{\mathrm{O}}>4\left(\sigma\left(\mathrm{F}_{\mathrm{O}}\right)\right)$.

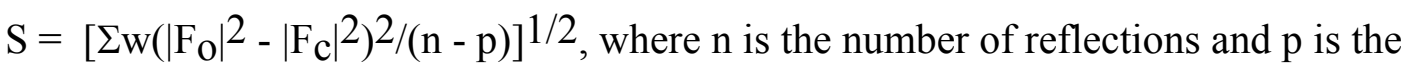
number of refined parameters.

20) International Tables for X-ray Crystallography (1992). Vol. C, Tables 4.2.6.8 and 6.1.1.4, A. J. C. Wilson, editor, Boston: Kluwer Academic Press.

6) Sheldrick, G. M. (1994). SHELXTL/PC (Version 5.03). Siemens Analytical Xray Instruments, Inc., Madison, Wisconsin, USA. 
Table 1. Crystal data and structure refinement for $\mathbf{9 .}$

Empirical formula

Formula weight

Temperature

Wavelength

Crystal system

Space group

Unit cell dimensions

Volume

Z

Density (calculated)

Absorption coefficient

$\mathrm{F}(000)$

Crystal size

Theta range for data collection

Index ranges

Reflections collected

Independent reflections

Completeness to theta $=27.50^{\circ}$

Absorption correction

Max. and min. transmission

Refinement method

Data / restraints / parameters

Goodness-of-fit on $\mathrm{F}^{2}$

Final $\mathrm{R}$ indices $[\mathrm{I}>2 \operatorname{sigma}(\mathrm{I})]$

$\mathrm{R}$ indices (all data)

Extinction coefficient

Largest diff. peak and hole
C42 H48 N4

608.84

153(2) K

$0.71073 \AA$

Triclinic

P-1

$\mathrm{a}=7.2910(2) \AA \quad \alpha=99.955(2)^{\circ}$.

$\mathrm{b}=10.8604(3) \AA \quad \beta=99.543(2)^{\circ}$.

$\mathrm{c}=12.4091(4) \AA \quad \gamma=108.311(2)^{\circ}$.

892.98(5) $\AA^{3}$

1

$1.132 \mathrm{Mg} / \mathrm{m}^{3}$

$0.066 \mathrm{~mm}^{-1}$

328

$0.20 \times 0.14 \times 0.14 \mathrm{~mm}^{3}$

2.03 to $27.50^{\circ}$.

$-9<=\mathrm{h}<=9,-14<=\mathrm{k}<=14,-16<=\mathrm{l}<=15$

6810

$4085[\mathrm{R}(\mathrm{int})=0.0264]$

$99.4 \%$

None

1 and 3891

Full-matrix least-squares on $\mathrm{F}^{2}$

4085 / 0 / 239

1.118

$\mathrm{R} 1=0.0522, \mathrm{wR} 2=0.1185$

$\mathrm{R} 1=0.0960, \mathrm{wR} 2=0.1358$

$3.9(6) \times 10^{-5}$

0.180 and -0.155 e. $\AA^{-3}$ 
Table 2. Atomic coordinates ( x 104) and equivalent isotropic displacement parameters $\left(\AA^{2} \mathrm{x}\right.$ $10^{3}$ ) for 9 . U(eq) is defined as one third of the trace of the orthogonalized Uij tensor.

\begin{tabular}{|c|c|c|c|c|}
\hline & $\mathrm{x}$ & $\mathrm{y}$ & $\mathrm{z}$ & $\mathrm{U}(\mathrm{eq})$ \\
\hline N1 & $3196(2)$ & $3027(1)$ & $3083(1)$ & $43(1)$ \\
\hline N2 & $8046(2)$ & $7357(1)$ & $5106(1)$ & $41(1)$ \\
\hline $\mathrm{C} 1$ & $4102(2)$ & $4008(2)$ & $3959(1)$ & $35(1)$ \\
\hline $\mathrm{C} 2$ & $5676(2)$ & $5229(1)$ & $4008(1)$ & $35(1)$ \\
\hline $\mathrm{C} 3$ & $6556(2)$ & $6181(1)$ & 4984(1) & $35(1)$ \\
\hline $\mathrm{C} 4$ & $3770(2)$ & $3070(2)$ & $2044(1)$ & $40(1)$ \\
\hline $\mathrm{C} 5$ & $5210(3)$ & $2551(2)$ & $1806(2)$ & $53(1)$ \\
\hline C6 & $5639(3)$ & $2504(2)$ & $750(2)$ & $57(1)$ \\
\hline $\mathrm{C} 7$ & $4703(3)$ & $2963(2)$ & $-58(1)$ & $53(1)$ \\
\hline $\mathrm{C} 8$ & $3260(3)$ & $3454(2)$ & $190(2)$ & $57(1)$ \\
\hline C9 & $2744(2)$ & $3501(2)$ & $1227(1)$ & $48(1)$ \\
\hline $\mathrm{C} 10$ & $6270(4)$ & 2049(3) & $2681(2)$ & $100(1)$ \\
\hline $\mathrm{C} 11$ & $5263(3)$ & $2927(2)$ & $-1181(2)$ & $76(1)$ \\
\hline $\mathrm{C} 12$ & $1130(3)$ & $4007(3)$ & $1476(2)$ & $86(1)$ \\
\hline $\mathrm{C} 13$ & $8754(2)$ & $7852(1)$ & $4200(1)$ & $36(1)$ \\
\hline C14 & $10024(2)$ & $7350(2)$ & $3694(1)$ & $42(1)$ \\
\hline $\mathrm{C} 15$ & $10703(3)$ & $7877(2)$ & $2826(1)$ & $46(1)$ \\
\hline $\mathrm{C} 16$ & 10196(3) & $8899(2)$ & $2481(1)$ & $48(1)$ \\
\hline $\mathrm{C} 17$ & $8966(3)$ & $9391(2)$ & $3020(1)$ & $47(1)$ \\
\hline $\mathrm{C} 18$ & $8193(2)$ & $8876(2)$ & $3873(1)$ & $41(1)$ \\
\hline C19 & $10668(3)$ & $6277(2)$ & $4070(2)$ & $60(1)$ \\
\hline $\mathrm{C} 20$ & 10924(3) & $9448(2)$ & $1524(2)$ & $70(1)$ \\
\hline $\mathrm{C} 21$ & $6768(3)$ & $9377(2)$ & $4401(2)$ & $55(1)$ \\
\hline
\end{tabular}


Table 3. Bond lengths $[\AA]$ and angles $\left[{ }^{\circ}\right]$ for $\mathbf{9}$.

\begin{tabular}{|c|c|c|c|}
\hline $\mathrm{N} 1-\mathrm{C} 1$ & $1.2931(19)$ & C11-H11B & 0.98 \\
\hline N1-C4 & $1.4241(18)$ & C11-H11C & 0.98 \\
\hline N2-C3 & $1.3569(19)$ & C12-H12A & 0.98 \\
\hline N2-C13 & $1.4302(18)$ & C12-H12B & 0.98 \\
\hline $\mathrm{N} 2-\mathrm{H} 2 \mathrm{~N}$ & $0.906(19)$ & C12-H12C & 0.98 \\
\hline $\mathrm{C} 1-\mathrm{C} 2$ & $1.440(2)$ & C13-C14 & $1.393(2)$ \\
\hline C1-C3\#1 & $1.4941(19)$ & $\mathrm{C} 13-\mathrm{C} 18$ & $1.399(2)$ \\
\hline $\mathrm{C} 2-\mathrm{C} 3$ & $1.356(2)$ & $\mathrm{C} 14-\mathrm{C} 15$ & $1.395(2)$ \\
\hline $\mathrm{C} 2-\mathrm{H} 2$ & $0.978(15)$ & C14-C19 & $1.502(2)$ \\
\hline C3-C1\#1 & $1.4941(19)$ & $\mathrm{C} 15-\mathrm{C} 16$ & $1.386(2)$ \\
\hline $\mathrm{C} 4-\mathrm{C} 5$ & $1.387(2)$ & C15-H15 & $0.994(18)$ \\
\hline C4-C9 & $1.392(2)$ & $\mathrm{C} 16-\mathrm{C} 17$ & $1.383(2)$ \\
\hline C5-C6 & $1.393(2)$ & $\mathrm{C} 16-\mathrm{C} 20$ & $1.518(2)$ \\
\hline C5-C10 & $1.505(3)$ & C17-C18 & $1.398(2)$ \\
\hline C6-C7 & $1.370(3)$ & $\mathrm{C} 17-\mathrm{H} 17$ & $1.009(17)$ \\
\hline C6-H6 & $0.97(2)$ & $\mathrm{C} 18-\mathrm{C} 21$ & $1.504(2)$ \\
\hline $\mathrm{C} 7-\mathrm{C} 8$ & $1.375(3)$ & C19-H19A & 0.98 \\
\hline C7-C11 & $1.514(2)$ & C19-H19B & 0.98 \\
\hline C8-C9 & $1.397(2)$ & C19-H19C & 0.98 \\
\hline C8-H8 & $1.03(2)$ & C20-H20A & 0.98 \\
\hline C9-C12 & $1.500(3)$ & C20-H20B & 0.98 \\
\hline C10-H10A & 0.98 & C20-H20C & 0.98 \\
\hline C10-H10B & 0.98 & $\mathrm{C} 21-\mathrm{H} 21 \mathrm{~A}$ & 0.98 \\
\hline C10-H10C & 0.98 & C21-H21B & 0.98 \\
\hline C11-H11A & 0.98 & $\mathrm{C} 21-\mathrm{H} 21 \mathrm{C}$ & 0.98 \\
\hline C1-N1-C4 & $120.34(12)$ & $\mathrm{C} 3-\mathrm{C} 2-\mathrm{H} 2$ & $120.7(9)$ \\
\hline C3-N2-C13 & $124.90(13)$ & $\mathrm{C} 1-\mathrm{C} 2-\mathrm{H} 2$ & $117.9(9)$ \\
\hline C3-N2-H2N & $113.6(12)$ & $\mathrm{C} 2-\mathrm{C} 3-\mathrm{N} 2$ & $125.25(13)$ \\
\hline C13-N2-H2N & $119.5(12)$ & $\mathrm{C} 2-\mathrm{C} 3-\mathrm{C} 1 \# 1$ & $120.59(13)$ \\
\hline $\mathrm{N} 1-\mathrm{C} 1-\mathrm{C} 2$ & $126.38(13)$ & N2-C3-C1\#1 & $114.16(13)$ \\
\hline N1-C1-C3\#1 & $115.56(12)$ & $\mathrm{C} 5-\mathrm{C} 4-\mathrm{C} 9$ & $120.18(14)$ \\
\hline C2-C1-C3\#1 & $118.06(13)$ & $\mathrm{C} 5-\mathrm{C} 4-\mathrm{N} 1$ & $119.58(14)$ \\
\hline $\mathrm{C} 3-\mathrm{C} 2-\mathrm{C} 1$ & $121.35(13)$ & C9-C4-N1 & $119.90(14)$ \\
\hline
\end{tabular}




\begin{tabular}{|c|c|c|c|}
\hline C4-C5-C6 & 118.77(17) & C13-C14-C15 & $118.21(15$ \\
\hline C4-C5-C10 & $119.92(16)$ & C13-C14-C19 & $121.49(14)$ \\
\hline C6-C5-C10 & $121.31(18)$ & C15-C14-C19 & $120.30(15)$ \\
\hline C7-C6-C5 & $122.38(18)$ & C16-C15-C14 & $122.01(16)$ \\
\hline C7-C6-H6 & $120.7(11)$ & $\mathrm{C} 16-\mathrm{C} 15-\mathrm{H} 15$ & $119.5(10)$ \\
\hline C5-C6-H6 & $116.9(11)$ & C14-C15-H15 & $118.4(10)$ \\
\hline C6-C7-C8 & $117.90(16)$ & $\mathrm{C} 17-\mathrm{C} 16-\mathrm{C} 15$ & $118.06(14)$ \\
\hline C6-C7-C11 & $120.39(18)$ & C17-C16-C20 & $120.54(16)$ \\
\hline C8-C7-C11 & $121.71(18)$ & $\mathrm{C} 15-\mathrm{C} 16-\mathrm{C} 20$ & $121.38(17)$ \\
\hline C7-C8-C9 & $122.08(18)$ & C16-C17-C18 & $122.48(16)$ \\
\hline C7-C8-H8 & $121.7(12)$ & C16-C17-H17 & $121.9(10)$ \\
\hline C9-C8-H8 & $116.2(12)$ & C18-C17-H17 & $115.6(10)$ \\
\hline C4-C9-C8 & $118.63(16)$ & $\mathrm{C} 17-\mathrm{C} 18-\mathrm{C} 13$ & $117.56(15)$ \\
\hline $\mathrm{C} 4-\mathrm{C} 9-\mathrm{C} 12$ & $119.83(15)$ & $\mathrm{C} 17-\mathrm{C} 18-\mathrm{C} 21$ & $121.44(15)$ \\
\hline C8-C9-C12 & $121.54(17)$ & $\mathrm{C} 13-\mathrm{C} 18-\mathrm{C} 21$ & $120.98(14)$ \\
\hline C5-C10-H10A & 109.5 & C14-C19-H19A & 109.5 \\
\hline C5-C10-H10B & 109.5 & C14-C19-H19B & 109.5 \\
\hline H10A-C10-H10B & 109.5 & H19A-C19-H19B & 109.5 \\
\hline C5-C10-H10C & 109.5 & C14-C19-H19C & 109.5 \\
\hline $\mathrm{H} 10 \mathrm{~A}-\mathrm{C} 10-\mathrm{H} 10 \mathrm{C}$ & 109.5 & H19A-C19-H19C & 109.5 \\
\hline H10B-C10-H10C & 109.5 & H19B-C19-H19C & 109.5 \\
\hline C7-C11-H11A & 109.5 & C16-C20-H20A & 109.5 \\
\hline C7-C11-H11B & 109.5 & C16-C20-H20B & 109.5 \\
\hline H11A-C11-H11B & 109.5 & $\mathrm{H} 20 \mathrm{~A}-\mathrm{C} 20-\mathrm{H} 20 \mathrm{~B}$ & 109.5 \\
\hline C7-C11-H11C & 109.5 & $\mathrm{C} 16-\mathrm{C} 20-\mathrm{H} 20 \mathrm{C}$ & 109.5 \\
\hline H11A-C11-H11C & 109.5 & $\mathrm{H} 20 \mathrm{~A}-\mathrm{C} 20-\mathrm{H} 20 \mathrm{C}$ & 109.5 \\
\hline H11B-C11-H11C & 109.5 & $\mathrm{H} 20 \mathrm{~B}-\mathrm{C} 20-\mathrm{H} 20 \mathrm{C}$ & 109.5 \\
\hline C9-C12-H12A & 109.5 & C18-C21-H21A & 109.5 \\
\hline C9-C12-H12B & 109.5 & C18-C21-H21B & 109.5 \\
\hline $\mathrm{H} 12 \mathrm{~A}-\mathrm{C} 12-\mathrm{H} 12 \mathrm{~B}$ & 109.5 & $\mathrm{H} 21 \mathrm{~A}-\mathrm{C} 21-\mathrm{H} 21 \mathrm{~B}$ & 109.5 \\
\hline C9-C12-H12C & 109.5 & $\mathrm{C} 18-\mathrm{C} 21-\mathrm{H} 21 \mathrm{C}$ & 109.5 \\
\hline $\mathrm{H} 12 \mathrm{~A}-\mathrm{C} 12-\mathrm{H} 12 \mathrm{C}$ & 109.5 & $\mathrm{H} 21 \mathrm{~A}-\mathrm{C} 21-\mathrm{H} 21 \mathrm{C}$ & 109.5 \\
\hline $\mathrm{H} 12 \mathrm{~B}-\mathrm{C} 12-\mathrm{H} 12 \mathrm{C}$ & 109.5 & $\mathrm{H} 21 \mathrm{~B}-\mathrm{C} 21-\mathrm{H} 21 \mathrm{C}$ & 109.5 \\
\hline C14-C13-C18 & $121.63(13)$ & & \\
\hline C14-C13-N2 & $120.16(13)$ & & \\
\hline C18-C13-N2 & $118.18(14)$ & & \\
\hline
\end{tabular}


Symmetry transformations used to generate equivalent atoms:

$\# 1-\mathrm{x}+1,-\mathrm{y}+1,-\mathrm{z}^{+} 1$ 
Table 4. Anisotropic displacement parameters $\left(\AA^{2} \times 10^{3}\right)$ for 9. The anisotropic displacement factor exponent takes the form: $-2 \pi^{2}\left[h^{2} a^{* 2} U^{11}+\ldots+2 h \mathrm{k}^{*} b^{*} U^{12}\right]$

\begin{tabular}{|c|c|c|c|c|c|c|}
\hline & $\mathrm{U}^{11}$ & $\mathrm{U}^{22}$ & $\mathrm{U}^{33}$ & $\mathrm{U}^{23}$ & $\mathrm{U}^{13}$ & $\mathrm{U}^{12}$ \\
\hline N1 & $50(1)$ & $40(1)$ & $30(1)$ & $7(1)$ & $17(1)$ & $3(1)$ \\
\hline N2 & $50(1)$ & $35(1)$ & $28(1)$ & $6(1)$ & $15(1)$ & $-1(1)$ \\
\hline $\mathrm{C} 1$ & $40(1)$ & $35(1)$ & 29(1) & $10(1)$ & 11(1) & $8(1)$ \\
\hline $\mathrm{C} 2$ & $42(1)$ & $35(1)$ & $28(1)$ & 11(1) & $14(1)$ & $9(1)$ \\
\hline $\mathrm{C} 3$ & $37(1)$ & $35(1)$ & $32(1)$ & 12(1) & $13(1)$ & $8(1)$ \\
\hline $\mathrm{C} 4$ & $45(1)$ & $35(1)$ & $30(1)$ & $5(1)$ & $15(1)$ & $-1(1)$ \\
\hline $\mathrm{C} 5$ & $62(1)$ & $56(1)$ & $49(1)$ & $20(1)$ & $25(1)$ & $20(1)$ \\
\hline C6 & $64(1)$ & $56(1)$ & $56(1)$ & 11(1) & $33(1)$ & $20(1)$ \\
\hline $\mathrm{C} 7$ & $52(1)$ & $58(1)$ & $34(1)$ & $0(1)$ & $18(1)$ & $0(1)$ \\
\hline $\mathrm{C} 8$ & 49(1) & $82(1)$ & $36(1)$ & $18(1)$ & $14(1)$ & $12(1)$ \\
\hline C9 & $44(1)$ & $61(1)$ & $36(1)$ & 12(1) & $15(1)$ & $9(1)$ \\
\hline $\mathrm{C} 10$ & $118(2)$ & $146(3)$ & $99(2)$ & $77(2)$ & $58(2)$ & $90(2)$ \\
\hline C11 & $71(1)$ & $98(2)$ & $41(1)$ & $1(1)$ & $28(1)$ & $5(1)$ \\
\hline $\mathrm{C} 12$ & $76(2)$ & $143(2)$ & $75(2)$ & $57(2)$ & $43(1)$ & $58(2)$ \\
\hline $\mathrm{C} 13$ & $42(1)$ & $33(1)$ & $27(1)$ & $7(1)$ & $12(1)$ & $2(1)$ \\
\hline $\mathrm{C} 14$ & $49(1)$ & $36(1)$ & $36(1)$ & $8(1)$ & $15(1)$ & $7(1)$ \\
\hline $\mathrm{C} 15$ & $54(1)$ & $40(1)$ & $40(1)$ & $6(1)$ & $23(1)$ & $6(1)$ \\
\hline $\mathrm{C} 16$ & $64(1)$ & $39(1)$ & $34(1)$ & $9(1)$ & 21(1) & $2(1)$ \\
\hline $\mathrm{C} 17$ & $60(1)$ & $39(1)$ & 41(1) & $16(1)$ & $14(1)$ & 11(1) \\
\hline $\mathrm{C} 18$ & $43(1)$ & $39(1)$ & $37(1)$ & $8(1)$ & $13(1)$ & $7(1)$ \\
\hline C19 & $77(1)$ & $57(1)$ & $58(1)$ & $22(1)$ & $31(1)$ & $30(1)$ \\
\hline $\mathrm{C} 20$ & $102(2)$ & $52(1)$ & $48(1)$ & $17(1)$ & $37(1)$ & $3(1)$ \\
\hline $\mathrm{C} 21$ & $55(1)$ & $56(1)$ & $56(1)$ & $16(1)$ & $19(1)$ & $20(1)$ \\
\hline
\end{tabular}


Table 5. Hydrogen coordinates ( $\left.\times 10^{4}\right)$ and isotropic displacement parameters $\left(\AA^{2} \times 10\right.$ 3 ) for 9.

\begin{tabular}{|c|c|c|c|c|}
\hline & $\mathrm{x}$ & $\mathrm{y}$ & $\mathrm{z}$ & $\mathrm{U}(\mathrm{eq})$ \\
\hline $\mathrm{H} 10 \mathrm{~A}$ & 7141 & 2805 & 3300 & 150 \\
\hline H10B & 7068 & 1586 & 2341 & 150 \\
\hline $\mathrm{H} 10 \mathrm{C}$ & 5291 & 1428 & 2971 & 150 \\
\hline H11A & 6669 & 3471 & -1061 & 114 \\
\hline H11B & 4444 & 3283 & -1655 & 114 \\
\hline $\mathrm{H} 11 \mathrm{C}$ & 5040 & 2002 & -1555 & 114 \\
\hline $\mathrm{H} 12 \mathrm{~A}$ & 210 & 3364 & 1780 & 129 \\
\hline H12B & 407 & 4117 & 781 & 129 \\
\hline $\mathrm{H} 12 \mathrm{C}$ & 1719 & 4870 & 2029 & 129 \\
\hline H19A & 10683 & 6345 & 4869 & 89 \\
\hline H19B & 12004 & 6387 & 3957 & 89 \\
\hline $\mathrm{H} 19 \mathrm{C}$ & 9737 & 5398 & 3627 & 89 \\
\hline $\mathrm{H} 20 \mathrm{~A}$ & 12142 & 9276 & 1445 & 105 \\
\hline $\mathrm{H} 20 \mathrm{~B}$ & 11199 & 10413 & 1686 & 105 \\
\hline $\mathrm{H} 20 \mathrm{C}$ & 9900 & 9010 & 822 & 105 \\
\hline $\mathrm{H} 21 \mathrm{~A}$ & 5516 & 8633 & 4289 & 82 \\
\hline $\mathrm{H} 21 \mathrm{~B}$ & 6513 & 10066 & 4048 & 82 \\
\hline $\mathrm{H} 21 \mathrm{C}$ & 7346 & 9756 & 5208 & 82 \\
\hline $\mathrm{H} 2$ & $6080(20)$ & $5362(14)$ & $3310(13)$ & $38(4)$ \\
\hline H17 & $8530(20)$ & $10126(18)$ & $2801(14)$ & $58(5)$ \\
\hline H15 & $11640(30)$ & $7537(17)$ & $2478(15)$ & $58(5)$ \\
\hline $\mathrm{H} 2 \mathrm{~N}$ & $8340(30)$ & $7925(19)$ & $5790(16)$ & $60(5)$ \\
\hline H8 & $2500(30)$ & $3810(20)$ & $-382(18)$ & $85(7)$ \\
\hline H6 & $6630(30)$ & $2117(19)$ & $602(16)$ & $71(6)$ \\
\hline
\end{tabular}


Table 6. Torsion angles $\left[{ }^{\circ}\right]$ for $\mathbf{9}$.

\begin{tabular}{|c|c|}
\hline C4-N1-C1-C2 & $2.1(2)$ \\
\hline C4-N1-C1-C3\#1 & $-176.88(13)$ \\
\hline $\mathrm{N} 1-\mathrm{C} 1-\mathrm{C} 2-\mathrm{C} 3$ & $-178.36(15)$ \\
\hline $\mathrm{C} 3 \# 1-\mathrm{C} 1-\mathrm{C} 2-\mathrm{C} 3$ & $0.6(2)$ \\
\hline $\mathrm{C} 1-\mathrm{C} 2-\mathrm{C} 3-\mathrm{N} 2$ & $179.97(14)$ \\
\hline $\mathrm{C} 1-\mathrm{C} 2-\mathrm{C} 3-\mathrm{C} 1 \# 1$ & $-0.6(2)$ \\
\hline C13-N2-C3-C2 & $8.2(2)$ \\
\hline C13-N2-C3-C1\#1 & $-171.26(14)$ \\
\hline C1-N1-C4-C5 & $90.49(19)$ \\
\hline C1-N1-C4-C9 & $-96.23(19)$ \\
\hline C9-C4-C5-C6 & $1.7(3)$ \\
\hline N1-C4-C5-C6 & $174.91(15)$ \\
\hline C9-C4-C5-C10 & $-178.16(19)$ \\
\hline $\mathrm{N} 1-\mathrm{C} 4-\mathrm{C} 5-\mathrm{C} 10$ & $-4.9(3)$ \\
\hline $\mathrm{C} 4-\mathrm{C} 5-\mathrm{C} 6-\mathrm{C} 7$ & $0.6(3)$ \\
\hline C10-C5-C6-C7 & $-179.6(2)$ \\
\hline C5-C6-C7-C8 & $-1.6(3)$ \\
\hline C5-C6-C7-C11 & $178.24(18)$ \\
\hline C6-C7-C8-C9 & $0.3(3)$ \\
\hline C11-C7-C8-C9 & $-179.47(17)$ \\
\hline C5-C4-C9-C8 & $-2.8(2)$ \\
\hline N1-C4-C9-C8 & $-176.04(15)$ \\
\hline C5-C4-C9-C12 & $177.41(18)$ \\
\hline N1-C4-C9-C12 & $4.2(2)$ \\
\hline C7-C8-C9-C4 & $1.8(3)$ \\
\hline C7-C8-C9-C12 & $-178.39(19)$ \\
\hline C3-N2-C13-C14 & $-78.3(2)$ \\
\hline C3-N2-C13-C18 & $103.98(18)$ \\
\hline C18-C13-C14-C15 & $-1.3(2)$ \\
\hline N2-C13-C14-C15 & $-178.98(14)$ \\
\hline C18-C13-C14-C19 & $178.28(15)$ \\
\hline N2-C13-C14-C19 & $0.6(2)$ \\
\hline $\mathrm{C} 13-\mathrm{C} 14-\mathrm{C} 15-\mathrm{C} 16$ & $1.8(2)$ \\
\hline C19-C14-C15-C16 & $-177.81(16)$ \\
\hline
\end{tabular}


C14-C15-C16-C17

C14-C15-C16-C20

C15-C16-C17-C18

C20-C16-C17-C18

C16-C17-C18-C13

C16-C17-C18-C21

C14-C13-C18-C17

N2-C13-C18-C17

C14-C13-C18-C21

N2-C13-C18-C21
$-0.4(3)$

$-179.12(16)$

$-1.4(3)$

177.25(16)

1.9(2)

$-176.54(16)$

$-0.5(2)$

177.27(14)

$177.96(15)$

$-4.3(2)$

Symmetry transformations used to generate equivalent atoms:

$\# 1-\mathrm{x}+1,-\mathrm{y}+1,-\mathrm{z}^{+1}$ 